\title{
Energy Coupling across Low-dimensional Contact Interfaces at the Atomic Scale
}

\author{
Yanan Yue ${ }^{1,2, a, ~}$, Jingchao Zhang ${ }^{3, a}$, Yangsu Xie ${ }^{4}$, Wen Chen ${ }^{1,2}$, Xinwei Wang ${ }^{4, *}$ \\ ${ }^{1}$ School of Power and Mechanical Engineering, Wuhan University, Wuhan, Hubei, 430072, China \\ ${ }^{2}$ State Laboratory of Hydraulic Machinery Transients, Ministry of Education, Wuhan, 430072, China \\ ${ }^{3}$ Holland Computing Center, University of Nebraska-Lincoln, Lincoln, NE, 68588, USA \\ ${ }^{4}$ Department of Mechanical Engineering, Iowa State University, Ames, IA, 50010, USA
}

\begin{abstract}
The miniaturization in energy devices and their critical needs for heat dissipation have facilitated research on exceptional thermal properties of novel low-dimensional materials. Current studies demonstrated the main challenge for solving thermal transport issues is the large thermal contact resistance across these low-dimensional material interfaces when they are either bundled together or supported by a substrate. A clear understanding of thermal transport across these atomic interfaces through experimental characterization or numerical simulation is important, but nontrivial. Due to instrumentation limit, only a few thermal characterization methods are applicable. Accordingly, many studies have been conducted by theoretical analysis and molecular dynamics to understand the physical process during this ultra-fast and ultra-small thermal transport. In this review, both experimental work and molecular dynamics studies on atomic-scale thermal contact resistance of low-dimensional materials (from zero- to two-dimensional) are reviewed. Challenges as well as opportunities in the study of thermal transport in atomic-layer structures are outlined. Considering the remarkable complexity of physical/chemical conditions, there is still a large room in understanding fundamentals of energy coupling across these atomic-layer interfaces.
\end{abstract}

\footnotetext{
${ }^{\mathrm{a}}$ These authors contribute equally;

*Corresponding authors: Y. Yue, Email: yyue@ whu.edu.cn, Tel: 86-27-68772268; X. Wang, E-mail: xwang3@iastate.edu, Tel: 1-515-294-8023.
} 


\section{Introduction}

The innovation in material science in the past two decades brought new insight into novel low-dimensional materials such as carbon nanotube (CNT) and graphene [1-3]. Specifically to thermal science, these materials are superior thermal conductors as validated in both experiments and simulations $[4,5]$ and regarded as the next generation semiconductor materials $[6,7]$. As the miniaturization continues, energy dissipation in nanoelectronics becomes a bottleneck that hinders storage density or computation speed $[8,9]$. Excellent thermal property of low-dimensional materials leads to a solution to these problems [10-12]. Taking graphene as an example, the two-dimensional structure makes it extremely difficult for direct industrial implementation [13]. Meanwhile, the limited thickness of graphene greatly affects thermal transport in the in-plane direction. When it is supported on a substrate, large amount of energy dissipates through the graphene-substrate interface [14]. In such case, the interfacial thermal resistance becomes the main factor determining thermal performance [14]. Therefore, characterization of interfacial thermal resistance and understanding energy transport across two-dimensional (2D) atomic-layer interfaces are important. Similar to graphene, the thermal conductivity of CNT is large. However, there are also thermal contact problems [15-17]. The thermal resistance of integrated CNT materials is mainly attributed to the contact among tubes or between the tube and substrate [18-20]. Aside from CNT and graphene, a lot of other low-dimensional materials, such as $h$ - $\mathrm{BN}$, silicene, $\mathrm{MoS}_{2}$, black phosphorus, etc., emerged in recent years. Therefore, the energy coupling across atomic-scale contacts related to these materials is of great interests and needs to be clarified with physical mechanism details.

Exploring such problem especially using experimental characterization is very challenging. As to CNT, the fabrication of specific CNT-CNT contact for characterization purpose is extremely difficult $[21,22]$, not to mention the limitation in 
experimental technologies and the complex physical conditions at the contact [22]. For 2D material-substrate interfaces, either conventional steady-state method or transient method has limitations for probing temperature at the atomic scale [23]. To achieve such task, researchers seek solutions from material modifications: for example, coating an additional layer on top of the 2D material to improve the thickness of measurement target and thus the conventional measurement method is applicable [24, 25]. However, the constraint effect brought by the additional layer results in different and undesirable phonon transport scenarios [26]. Meanwhile, the corrugation or wrinkling of 2D materials further increases the complexity of experiment [26, 27]. Classical molecular dynamics (MD) simulations have been widely used in exploring such problem beyond the scope of first principle calculations [28].

In this paper, the state-of-the-art studies about the energy coupling across atomic-scale interfaces are reviewed to uncover challenges, propose strategies to tackle tough technical challenges, and provide guidance on the applicability of current technologies in characterization atomic scale interface energy transport. For distinguishing the physical mechanism, the thermal contact resistance will be termed in zero-dimensional and one-dimentional thermal contact analysis; as to 2D interfaces such graphene interfaces, the interfacial thermal resistance/conductance will be termed for better understanding.

\section{Physics and techniques applied for measurement}

Thermal transport across atomic scale interface is an intriguing problem. Taking the graphene-substrate thermal contact as an example, the thickness of graphene is only in order of sub-nm or $\sim \mathrm{nm}$. The physical definition of interfacial thermal resistance becomes debatable. As from experimental aspect, thermal characterization requires highly sensitive temperature probing and accurate heat flux management techniques. Finding effective ways to apply heat current and measure temperature difference across 
the atomic-scale interface is critical and extremely challenging. The techniques used to date are reviewed below to analyze their advantages, drawbacks, and provide strategies on how to improve.

\subsection{Thermal contact of zero- to one-dimensional nanostructures}

The extremely small contact area of 1D nanoscale materials, either with substrates or among them leads to the fact that thermal resistance at contacts dominates heat dissipation in bulk materials [20]. For instance, the cross alignment of CNTs forms zero-dimensional (point) contact. Similar scenarios include the contacts between particle/particle, particle/substrate, and CNTs vertically standing on a substrate, etc. For parallel aligned CNT arrays, thermal contact between CNTs becomes $1 \mathrm{D}$, similar to a lateral CNT lying on a substrate. The experimental characterization of interfacial thermal conductance for any of these structures is very challenging, not only because of its tiny size that increases the difficulty in sample fabrication, but also because of the complexity in different contact scenarios.

Systematic work has been first reported by Li's group [21]. They measured the thermal contact resistance between multiwall CNTs by using the classical micro-bridge method $[29,30]$. Two CNTs are interacted with each end attaching on different membranes as shown in Fig. 1(a) for a cross-contact and an aligned CNT contact [21]. Thermal transport of the system follows 1D heat conduction model and the total thermal resistance is contributed from each tube and the contact resistance between them. To determine the thermal contact resistance, the thermal conductivity of each tube needs to be determined in advance. Besides the micro-bridge method, a contact transient electro-thermal (CTET) technique is also available to characterize point contact thermal resistance to the nanoscale. The CTET technique has been developed and demonstrated by measuring the thermal contact resistance between microwires [31]. As shown in Fig. 1(b), two 
micro/nanoscale wires are suspended on two pairs of electrodes and cross-contacted by adjusting sample holders. During the measurement, the top wire is heated. The heat dissipates from the hot wire to the bottom one through their contact point. There is a temperature drop at this contact, which can be used for thermal contact resistance measurement. The thermal property of each wire also needs to be evaluated in advance which can be achieved by using the transient-electro-thermal (TET) technique before the two wires are in contact [32]. Different from Yang et al.'s work that the thermal conductivity of CNTs is determined from the parallel measurement of a similar nanotube [21], thermal property of the same wire can be precisely determined in CTET method. To apply the CTET technique to nanowire contact, very fine positioning of suspended nanowires are needed.

The thermal contact resistance between a 1D nanostructure and its substrate is commonly encountered in engineering applications. This 1D thermal contact resistance is almost impossible to measure. For a 1D thermal contact formed by a lateral single-walled CNT and insulating substrate, Pop et al. did the measurement based on thermally induced break down of CNTs [33]. As shown in Figure 1(c), the ends of a CNT are connected to Pt electrodes for Joule heating. The Joule heat in CNT dissipates through contact to the substrate. If length and diameter of the CNT sample are obtained, the heating density is calculated from breakdown current of CNT [34]. As breakdown temperature of CNT is determined and contact area is estimated from the CNT diameter, the thermal contact resistance per unit area from CNT to the insulating substrate can be calculated from the temperature difference (across the interface). Another example focusing on the 1D contact resistance is performed in Shi's lab for probing temperature profile along Joule-heated multi-wall and single-wall CNTs on a substrate [35]. The interface thermal conductance between CNT and substrate is evaluated based on an estimated thermal conductivity of CNTs [35]. Another scenario for 1D thermal contact is the array where 
the tubes are well aligned, and the 1D contact is between similar materials. Thermal characterization along the axial direction is commonly undertaken, especially for thermal interface materials analysis [36]. However, besides aforementioned work for the parallel contact formed by two CNTs [21], very limited work has been reported on the inter-1D material thermal contact resistance, mainly due to the great limitation of measurement techniques. Guo et al. characterized inter-tube thermal contact resistance of $\mathrm{TiO}_{2}$ nanotube arrays by combining the TET technique and photothermal technique [37]. Thermal conductivity of $\mathrm{TiO}_{2}$ arrays in the inter-tube direction is measured first. The measured value is a combined effect of thermal conductance in the wall of $\mathrm{TiO}_{2}$ tube and thermal contact conductance between tubes, which dominates thermal transport in the sample because of the large amount of contacts along the cross-nanotube heat conduction direction. The thermal contact resistance is extracted by considering two thermal transport directions ( $\alpha$ path and $\beta$ path) as shown in Figure 1(d) [37].

As a general rule, when studying the thermal contact resistance between 1D nanostructures, the thermal conductivity or resistance of the $1 \mathrm{D}$ nanostructure has to be measured first, either along the axial direction or in the radial direction. This is an essential part in the characterization. Due to limited/unknown knowledge of the contact area, it is very difficult to give a thermal contact resistance per unit area. Instead, a thermal contact resistance per unit length of the 1D nanostructure is more accurate and physically preferred. For measuring the thermal contact resistance between 1D nanostructure and its substrate, the thermal conductivity of 1D nanostructure usually is not needed or has insignificant effect on the measurement. However, the contact area between 1D nanostructures or between a 1D nanostructure and its substrate can vary a lot during sample preparation, and will lead to significant variation of the thermal contact resistance. This raises great challenges in evaluating the measurement accuracy of different techniques since no standard samples are available. 


\subsection{Constrained 2D atomic-layer thermal contact: challenges solved by additional material/transducer layer}

2D thermal contact resistance can be regarded as an extreme case of thermal transport in the thickness direction of atomic structures. Aside from complex surface morphology, the interface thermal characterization is challenging due to the atomic scale material thickness, which theoretically requires the same-level of spatial resolution for thermal characterization [38]. Either conventional steady-state method or transient method has limitations for probing temperature at such small scales: steady-state method either based on thermoelectric effect (for example the thermocouple) or laser excitation is impossible to measure the temperature of monolayer atoms. Hence, probing the material temperature is the main obstacle.

In transient methods, the time constant for thermal relaxation is the key factor. Taking transient laser reflectance technique as an example, most of the probing laser would penetrate graphene to substrate and the laser reflectance is mostly from the substrate. Thus, the measured temperature is a combined value of graphene and substrate surface and unable to distinguish them. Besides, the characteristic time for heat conduction across the graphene interface is extremely short due to its tiny thickness. It can be estimated as $\tau=\rho V c /(G A)$ where $\rho$ is density of graphene, $V$ its volume, $A$ the surface area, $c$ its specific heat, and $G$ the effective thermal conductance (the inverse of interfacial thermal resistance). With an estimation based on the heat capacity of graphite, and assuming the interfacial thermal resistance in the order of $10^{-9} \mathrm{~K} \cdot \mathrm{m}^{2} / \mathrm{W}$ (for a tight contact), $\tau$ of graphene is in the order of $10^{-13} \mathrm{~s}(100 \mathrm{fs})$, which is beyond the capacity of most measurement techniques for monitoring temperature with such short response, unless the ultra-fast laser technology is employed [23].

As mentioned above, researchers seek solutions by coating an additional layer on top of 
2D atomic layer materials to increase the thermal mass and reduce the thermal relaxation time $[24,25]$. However, the material modification might introduce extra problems. For example, the constraint effect brought by the additional layer results in a different phonon transport [26]. The scenario is complicated for $2 \mathrm{D}$ material interfaces with chemical residues or functional groups [25,39], or with different atomic potentials: covalent bonds [40] and van der Waals bond [39]. Also the additional tranducer layer will eliminate or greatly reduce the corrugation or wrinkling of $2 \mathrm{D}$ atomic materials $[26,27]$. The measured thermal contact resistance could not reflect the virgin or intrinsic $2 \mathrm{D}$ atomic layer material interface.

\subsubsection{Thermal probing in the frequency domain}

After the $2 \mathrm{D}$ atomic layer material is covered by a top layer, the thermal relaxation time of the sample becomes much larger and more measurable in both time and frequency domains. For frequency domain methods, the sample is thermally excited/heated periodically with its temperature oscillating in accordance with the heating source. The phase shift and temperature response amplitude are tightly related to the thermophysical properties of the material, such as thermal conductivity, thermal diffusivity, and thermal resistance. The most widely used frequency domain methods include photo-acoustic/thermal method and $3 \omega$ method [41, 42]. The photo-acoustic/thermal techniques are classical and have been well applied to thermal transport measurement of nanostructured interfaces [36], thin films [43] and bulk materials [44]. Frequency domain thermo-reflectance (FDTR) is the most commonly used frequency domain method in thermal characterization of 2D material interfaces. It shares the same physical and experimental principle as the photo-thermal and photo-acoustic technique, but uses a second probe beam to measure the surface thermal response by measuring the reflectance change [45]. Yang et al. used FDTR to create thermal conductance maps of graphene contacts and obtain cross-plane thermal boundary conductance for 1-7 graphitic layers 
sandwiched between titanium and silicon dioxide as shown in Figure 1(e) [46]. Majumdar et al. measured the thermal conductance of self-assembled monolayer junctions formed between metal leads ( $\mathrm{Au}, \mathrm{Ag}, \mathrm{Pt}$, and $\mathrm{Pd}$ ) [47]. Jeong et al. measured the interfacial thermal conductance across $\mathrm{Au}$ and $\mathrm{Al}_{2} \mathrm{O}_{3}$ interface with adhesion $\mathrm{Cu}$ and $\mathrm{Cr}$ metal layers by using FDTR method and found that $\mathrm{Cu}$ layer and $\mathrm{Cr}$ layer can improve the interfacial thermal conductance by a factor of 2 or 4 than that of the pure $\mathrm{Au} / \mathrm{Al}_{2} \mathrm{O}_{3}$ interface [48].

In the above laser-based frequency-domain techniques, a laser is employed as the heating source and is modulated within a limited frequency range. Therefore there is a thickness requirement in the target material for light absorption and thermal wave propagation. Photo-acoustic/thermal techniques might not be capable of measuring the thermal transport across atomic-scale (sub-nm) interfaces unless ultra-fast modulation techniques become available or by coating a metallic layer on top. Since laser is absorbed to generate heat, the top metallic layer has to be thick enough to absorb the laser completely. Otherwise, not-well defined laser absorption will greatly affect the measurement accuracy. Such thick layer requirement can be solved by using Joule heating. It is worth pointing out that the FDTR technique can achieve a higher modulation frequency and higher spatial resolution than the photo-thermal and photo-acoustic techniques.

The $3 \omega$ method is a Joule-heating method in frequency domain first reported by Dr. David Cahill [49]. In this technique, a very thin metallic wire is deposited on the sample surface serving as a heating source as well as the temperature sensor. An AC current with a frequency of $\omega$ is applied to the wire. The heat flux and corresponding temperature rise experience an oscillation with a frequency of $2 \omega$, which changes the electrical resistance of the wire in the same frequency. To the end, a voltage variation of $3 \omega$ frequency will arise, which can be used to determine thermal properties of a material $[50,51]$. It is 
required for the application of the $3 \omega$ method that the thermal penetration length should be much larger than heater line's width. Therefore, for samples with sub-nm thickness, graphene interfaces as an example, the $3 \omega$ method is not applicable unless a sub-nm heater line can be fabricated or an extremely high modulation frequency is used, which would outrange the capacity of current available instruments. Another problem is that graphene is a conductive material, which is not suitable to deposit the heating wire directly on it. An alternative approach is to increase thermal penetration depth by depositing an additional oxide layer on the to-be-measured nanoscale interfaces. Borca-Tasciuc et al. proposed a modified $3 \omega$ technique based on a differential method. It can be understood as a controlled-condition experiment: the difference in temperature rise between a film-substrate system and a same substrate system but without the film is measured, and the difference can be counted for the interface thermal resistance [50]. Chen et al. applied this method to measure the thermal contact resistance of sandwiched graphene- $\mathrm{SiO}_{2}$ interfaces [24].

\subsubsection{Measurement in the time domain}

Different from the frequency domain methods which use the phase shift and thermal response amplitude, time domain methods utilize the time scale information (e.g. the time constant of thermal relaxation) of the thermal transport inside the material to tackle thermophysical problems. As long as laser heating is used, these techniques suffer the same drawbacks of the laser-based frequency-domain techniques discussed above. One typical technique is the time domain thermal reflectance (TDTR or called pump-probe) method [42]. In the TDTR method, the heating source is an ultra-fast pulsed laser. When heated by the laser beam, the sample experiences a fast temperature rise due to the laser absorption and a gradual temperature drop due to heat dissipation [52-54]. A separate beam from the pump laser but with much less energy is used to monitor temperature based on the variation in reflectivity which is temperature dependent. 
As analyzed above, coating of a metallic layer is necessary to increase the thermal relaxation time and to achieve well-defined laser energy absorption and surface temperature probing. Using TDTR, Mak et al. reported the interface thermal conductance between exfoliated graphene and $\mathrm{SiO}_{2}$ in 2010 [55]. Koh et al. studied the overall heat conductance of multilayer graphene metal interfaces [56]. Hopkins et al. brought the chemical functionalization into interfacial thermal transport study of graphene metal interfaces [39]. Zhang et al. compared the interfacial thermal conductance of $\mathrm{Al} /$ graphene/Si structure with Al/Si interface (no graphene embedded, as shown in Fig. 1f) to study the effect of inserted graphene on interfacial thermal transport between metal and non-metal interfaces [25]. Vasquez Guzman et al. investigated the interfacial thermal conductance of monolayer graphene with different adhesion metal interfaces [57]. Most of above referenced work reported the overall thermal conductance of graphene/metal interfaces rather than distinguishing contact resistance of graphene with other materials except Zhang et al.'s work. To precisely determine the 2D material thermal contact resistance, the thermal conductivity of the top coating layer has to be measured. Also the thermal contact resistance of other interfaces in the structure needs to be determined. All of these are feasible, but nontrivial. Also determination of these extra parameters will bring in extra and significant uncertainty in the final measurement results.

\subsection{Unconstrained $2 \mathrm{D}$ atomic-layer thermal contact}

In many applications, bare supported 2D material (without coating) is preferred. Therefore, coating the 2D material with an extra layer to facilitate the measurement becomes very undesirable. As the sole steady state method, Raman thermometry can directly characterize interfaces of bare 2D materials $[4,58]$. Different characteristics of Raman spectrum properties can be used as the temperature indicator: Raman peak shift (frequency, or called wavenumber), peak intensity and peak linewidth. Among them, 
Raman peak shift is very sensitive to temperature change and most widely used. For example, the G-band peak of graphene was calibrated with a temperature coefficient of $\sim 0.016 \mathrm{~cm}^{-1} / \mathrm{K}[59]$. It needs to be pointed out that peak shift can be affected by stress induced strain effect. Peak intensity is temperature dependent but is easily affected by the focal level of optical path. Besides peak shift and intensity, the linewidth of Raman peak is independent on strain effect [14]. The disadvantage is that its sensitivity is not as high as peak shift. However, it provides a solution to measure thermal stress by combining the analysis of peak shift [26]. For the implementation of Raman thermometry for interfacial thermal characterization, the top layer of the structure needs to be thin enough for excitation laser to penetrate. Scattered Raman signal contains simultaneous temperatures of both substrate and film under a certain heating. Specifically, Raman thermometry for interface thermal characterization can be divided into two methods from heating source: joule (electrical) heating method and photon (laser) heating method.

\subsubsection{Joule heating Raman probing method}

As shown in Figure 2(a), the graphene supported on a substrate is connected by electrodes and heated by a constant current. The temperature difference across interface depends on heating density and interfacial thermal resistance. For monolayer graphene which absorbs $2.3 \%$ and reflects less than $0.1 \%$ of laser energy [60], most of light can penetrate and reach the substrate. Excited Raman signal contains temperature information for both graphene and substrate showing as distinctive Raman peaks in one spectrum. Interfacial thermal resistance is calculated according to the applied heating density [14]. Joule heating is uniform and can be well controlled for different experimental conditions. The disadvantages are obvious: first, joule heating requires the fabrication of electric circuit; second, the additional heating from the probe laser needs to be carefully treated during Raman measurement. However, these problems can be mitigated to the minimal level. Joule heating Raman probing method is first applied on interfacial thermal 
characterization of epitaxial graphene on $\mathrm{SiC}$ by Yue et al. [14]. It is noticed that the measured temperature of $\mathrm{SiC}$ is not from the surface but an average value within laser penetration depth [14]. The portion of Raman signal from different layer along laser penetration is not constant. A calibration needs to be performed to evaluate surface temperature of $\mathrm{SiC}$.

\subsubsection{Photon heating Raman probing measurement}

Photon (laser) heating is an alternative option to induce temperature difference for interfacial thermal characterization. It avoids the micro/nanofabrication of electrical circuit, being capable of probing samples with extremely small size. However, it must be pointed out that in photon heating, the optical properties of the $2 \mathrm{D}$ materials must be known. Even for the same 2D material, there are many scattered data in literatures for the optical properties, and large deviations exist among the data. These could introduce very large uncertainty in calculating the laser beam absorption in the $2 \mathrm{D}$ material. Also in experiment using a microscope objective, the laser beam is focused with a finite numerical aperture. Therefore, precise calculation of the laser beam absorption considering different incident angles is critical, but difficult to implement. On the other hand, the joule heating method can provide much reliable and well-defined heating data.

In Joule heating method, if the 2D material is not uniform in space, heating density will not be uniform. Also for 2D materials of very large electrical resistance, it is very difficult to apply Joule heating. In the photon heating method, the heating region and the Raman probing region are the same, which improves the measurement accuracy significantly. Compared with the 2D material thickness, the size of laser beam is much larger and the heat conduction across the 2D material interface can be regarded as $1 \mathrm{D}$. Cai et al. applied the photon heating method to measure thermal contact resistance between graphene and substrate [61]. In their work, only the graphene temperature 
instead of temperature difference across the interface was measured. Tang et al. employed two lasers to apply this technique: the higher energy laser is used for heating and the lower one is used for Raman probing [26]. Figure 2(b) shows that the probing area needs to be smaller than the heating area. This unique design for photon-heating and Raman probing effectively avoided any optical alignment shift due to the objective lens change in confocal Raman. Our past experience confirms that a small change in the optical alignment can introduce undesired shift in Raman spectrum, including its wavenumber, linewidth, and intensity. In the separate photon heating method, the Raman system stays at the exactly same configuration, and only the power of the heating laser is varied. In Tang's work, the temperatures of graphene and Si are measured simultaneously, and the temperature difference across the interface is directly probed. This provides much higher accuracy in determining interface thermal property. Using the similar design, Tang et al. also characterized the interfacial thermal resistance of graphene/SiC interface. For single-laser beam heating and Raman probing method, caution should be exercised for using different objectives or neutral density filter to adjust the Raman excitation laser energy to vary the heating level. In summary, although Raman spectroscopy can measure the temperature differential across the 2D material interface, precise control and determination of the heating level are key factors in affecting the measurement accuracy. Joule heating and photon heating each has its unique feature and drawback, and should be chosen based on material type and experiment control capacity.

\section{Thermal resistance/conductance of interfaces: variation and understanding}

As the interaction scenario between adjacent materials varies, the interfacial thermal transport related to these atomic scale materials is complicated and hard to compare. Even for the same material, variation in the sample preparation will significantly affect the interface structure, and greatly changes the interface thermal performance. Additionally, for the reported data in literature, some have large measurement uncertainty 
and are difficult to pin down the intrinsic interfacial thermal property. These could explain the very large data deviation reported in literatures. In review of these data, our focus is trying to reveal/understand these measurement values rather than comparing them. Selected works are summarized in Table 1.

\subsection{Zero-to-one dimensional thermal contacts}

The CNT-CNT contact features the most critical scenario, transforming from point to 1D contact with decreased angle between them. Yang et al. measured the thermal resistance of a cross contact between a $74 \mathrm{~nm}$ and a $121 \mathrm{~nm}$ (in diameter) nanotube, and an aligned contact of a $170 \mathrm{~nm}$ and a $165-185 \mathrm{~nm}$ (in diameter) nanotube [21]. It is found that thermal contact resistance is in the order of $10^{7} \mathrm{~K} / \mathrm{W}$ for both across contact and aligned contact. The contact resistance per unit area was calculated by counting the contact area (obtained from MD simulations) as $1.2 \times 10^{-8} \mathrm{~K} \cdot \mathrm{m}^{2} / \mathrm{W}$ for aligned contact and $1.22 \times 10^{-9}$ $\mathrm{K} \cdot \mathrm{m}^{2} / \mathrm{W}$ for the cross contact. This work is the first experimental report of thermal contact resistance between CNTs [21]. Their group reported another finding about the contact resistance between CNTs: the interface thermal conductance is a function of tube diameter (the thickness) ranging from $3 \times 10^{8}$ to $13 \times 10^{8} \mathrm{~W} / \mathrm{K} \cdot \mathrm{m}^{2}$ for different diameters with temperature from 50 up to $400 \mathrm{~K}$ [22]. They explained this abnormal thickness dependent nanoscale thermal contact via longer phonon mean free path in the $c$-axis direction of graphite, phonon reflection and focusing effect [22]. Velson et al. reported thermal contact resistance between crossed $\mathrm{Pt}$ wires as $8.94 \times 10^{4} \mathrm{~K} / \mathrm{W}$ to $7.05 \times 10^{5} \mathrm{~K} / \mathrm{W}$ under different heating currents from $20 \mathrm{~mA}$ to $50 \mathrm{~mA}$. In their work, the diameter of the Pt wires is $25.4 \mu \mathrm{m}$, and the contact resistance per unit area was estimated to be $10^{-7}-10^{-5}$ $\mathrm{K} \cdot \mathrm{m}^{2} / \mathrm{W}$ by assuming contact area of $1-10 \mu \mathrm{m}^{2}$ [31]. They also measured the contact resistance between a grass fiber $(8.9 \mu \mathrm{m})$ and $\mathrm{Pt}$ wire as $2.83 \times 10^{6} \mathrm{~K} / \mathrm{W}$, much larger than that of the Pt-Pt contact. The larger value is attributed to the smaller size of the glass fiber and the smaller contact area. 
For 1D thermal contact, Pop et al. reported the thermal conductance between metallic CNTs and insulating substrate as $0.17 \pm 0.03 \mathrm{~W} / \mathrm{m} \cdot \mathrm{K}$ [33]. By assuming the contact area as the product of length and diameter, the thermal contact resistance is estimated as $1-2 \times 10^{-8}$ $\mathrm{K} \cdot \mathrm{m}^{2} / \mathrm{W}$ [33]. Similar work by Maune et al. presented a thermal conductance per unit length about $0.33 \mathrm{~W} / \mathrm{m} \cdot \mathrm{K}$ between single-walled $\mathrm{CNT}$ and sapphire substrate [62]. The thermal resistance (per unit length) between aligned $\mathrm{TiO}_{2}$ nanotube arrays was measured as 15.1 and $20.6 \mathrm{~K} \cdot \mathrm{m} / \mathrm{W}$ for two amorphous samples, and $5.90 \mathrm{~K} \cdot \mathrm{m} / \mathrm{W}$ for anatase structure [37]. Considering the small contact area per unit length, which is in the order of $10^{-8} \mathrm{~m}^{2}$, the thermal contact resistance per unit area was determined in the order of $10^{-7}$ $\mathrm{K} \cdot \mathrm{m}^{2} / \mathrm{W}$ [37]. The thermal conductance between single wall CNT and substrate is measured to be in the range of $0.007-0.06 \mathrm{~W} / \mathrm{m} \cdot \mathrm{K}$ [35], which is much smaller than the value obtained by aforementioned electrical breakdown method. They attributed the difference to measurement temperature since the interface resistance is related to heat capacity which is temperature dependent. For all the reported work here, it is extremely difficult to give sound value of the thermal contact resistance/conductance per unit area as the contact area is unknown. Therefore, the thermal resistance/conductance per unit length or per contact point should be used and quoted to reflect the true scenario.

\subsection{Constrained 2D atomic-layer interfacial thermal transport}

\subsubsection{Experimental results measured with frequency-domain method}

Interfacial thermal resistance of graphene- $\mathrm{SiO}_{2}$ measured by the differential $3 \omega$ method ranges from $5.6 \times 10^{-9}$ to $1.2 \times 10^{-8} \mathrm{~K} \cdot \mathrm{m}^{2} / \mathrm{W}$ with temperatures from 42 to $310 \mathrm{~K}$ [24]. In this work, graphene flakes of $1.2 \mathrm{~nm}$ to $3 \mathrm{~nm}$ thickness are sandwiched between $\mathrm{SiO}_{2}$ layers. The measured contact resistance is close to the normal value for a tight contact (in

the order of $10^{-9} \mathrm{~K} \cdot \mathrm{m}^{2} / \mathrm{W}$ ), which can be understood that coating of oxide layers make graphene interface much tighter. Thermal transport across graphene-substrate interfaces (on both sides of graphene) is not the sole channel for heat dissipation. Phonon 
transmission between two oxide layers is possible. Since graphene is so thin that the distance between coated layer and substrate might be still within the atomic potential influence range. Yang et al. used the FDTR method to measure cross-plane thermal boundary conductance for 1-7 graphene layers encased between titanium and silicon dioxide. The thermal conductance of single-layer graphene/ $\mathrm{SiO}_{2}$ interface is obtained as $42 \mathrm{MW} / \mathrm{m}^{2} \cdot \mathrm{K}$ [46]. Majumdar et al. measured the thermal conductance of self-assembled monolayer junctions formed between metal leads ( $\mathrm{Au}, \mathrm{Ag}, \mathrm{Pt}$, and $\mathrm{Pd}$ ) by using FDTR method, and thermal conductance are measured as $65 \mathrm{MW} / \mathrm{m}^{2} \cdot \mathrm{K}$ for matched $\mathrm{Au}$-alkanedithiol-Au junctions, and $36 \mathrm{MW} / \mathrm{m}^{2} \cdot \mathrm{K}$ for mismatched $\mathrm{Au}-$ alkanedithiol-Pd junctions [47]. It is found that thermal conductance (per molecule) decreases as the mismatch between the lead vibrational spectra is increased. They explained that the junction thermal conductance would decrease because the number of overlapping vibrational modes between the self-assembled monolayer and the two leads would decrease when the mismatch between the lead vibrational spectra increases. Jeong et al. measured thermal conductance of $\mathrm{Au} / \mathrm{Al}_{2} \mathrm{O}_{3}$ interface with $\mathrm{Cu}$ and $\mathrm{Cr}$ adhesion layer and obtained saturated values of interfacial thermal conductance as $180 \mathrm{MW} / \mathrm{m}^{2} \cdot \mathrm{K}$ for $\mathrm{Cu}$ adhesion layer and $390 \mathrm{MW} / \mathrm{m}^{2} \cdot \mathrm{K}$ for $\mathrm{Cr}$ adhesion layer as adhesion layer thickness exceeded $5 \mathrm{~nm}$ [48].

\subsubsection{Experimental results measured with time-domain method}

The same issue brought by extra coating also exists in the TDTR method $[25,39,56]$. The effect of additional metallic layer on thermal transport across atomic layer interface is an interesting problem to explore. Zhang et al. embedded graphene between thermally evaporated $\mathrm{Al}$ film and $\mathrm{Si}$ substrate and found that this configuration could facilitate excellent interfacial thermal transport, showing an apparently negative interfacial thermal resistance between graphene and interface materials [25]. It is explained that graphene prevents the diffusion of $\mathrm{Au}$ atoms into substrate and reduces the thickness of intermixing 
layer. They also conducted measurement of magnetron sputtered $\mathrm{Al}$ films, and found that the embedded graphene contributes to the interfacial thermal resistance for magnetron sputtered Al film (for the increased number of interfaces) [25]. The results prove that different coatings on graphene layer affect interfacial thermal transport significantly. Koh et al. conducted the TDTR experiment to measure the overall thermal conductance of $\mathrm{Au} / \mathrm{Ti} / \mathrm{graphene} / \mathrm{SiO}_{2}$ interface as $25 \mathrm{MW} / \mathrm{m}^{2} \cdot \mathrm{K}$ which is much smaller than that of the $\mathrm{Au} / \mathrm{Ti} / \mathrm{SiO}_{2}$ interface [56]. They attribute the reduction in phonon transmission to the limit in graphene/metal contact [56].

Hopkins et al. measured thermal conductance of $\mathrm{Al} / \mathrm{graphene} / \mathrm{SiO}_{2}$ from 20 to 30 $\mathrm{MW} / \mathrm{m}^{2} \cdot \mathrm{K}$ for temperatures from $100 \mathrm{~K}$ to $400 \mathrm{~K}$ [39]. They found the hydrogen functionalization process introduced disorder in graphene while oxygen functionalization improved covalent bond between $\mathrm{Al}$ and graphene [39]. Foley et al. obtained thermal conductance of $\mathrm{Au} / \mathrm{O} /$ graphene interface from 24.33 to $32.41 \mathrm{MW} / \mathrm{m}^{2} \cdot \mathrm{K}$ for functionalization with oxygen coverage from $4.4 \%$ to $14.6 \%$, and Au/N/graphene interface from 21.41 to $28.98 \mathrm{MW} / \mathrm{m}^{2} \cdot \mathrm{K}$ for functionalization with nitrogen coverage from $1 \%$ to $13.2 \%$ [63]. It is reported that oxygen and nitrogen functionalization both increase surface energy to improve the surface reactivity of graphene, thus strengthen the contact between Au layer and graphene [63]. Koh et al. measured thermal conductance of $\mathrm{SiO}_{2} /$ graphene/ $/ \mathrm{SiO}_{2}$ interfaces under modulated electrostatic fields and found that the thermal conductance of graphene interfaces was increased by up to $\sim 0.8 \mathrm{MW} / \mathrm{m}^{2} \cdot \mathrm{K}$ under a vertical electrostatic field of $0.2 \mathrm{~V} / \mathrm{nm}$ by using a voltage-modulated thermoreflectance (VMTR) technique [64]. Two possible reasons are proposed for the enhancement: better conformity of graphene interfaces under electrostatic pressure exerted by the induced charge carriers, and an additional heat transfer channel by remote interfacial phonons scattering of charge carriers in graphene. 
Jiang et al. functionalized graphene with oxygen to investigate the interfacial thermal conductance between graphene and $\mathrm{Cu}, \mathrm{Al}$, and $\mathrm{Pt}$ under different degree of graphene oxidation [65]. It is found that thermal conductance is increased with oxidation coverage until a peak value $(\sim 7.7 \%)$, and about 95,87 and $105 \mathrm{MW} / \mathrm{m}^{2} \cdot \mathrm{K}$ for interfaces with $\mathrm{Cu}$, $\mathrm{Al}$, and $\mathrm{Pt}$, respectively. The maximum enhancement is $55 \%, 38 \%$, and $49 \%$ over corresponding original interfaces respectively. It is explained that oxygen atoms form covalent bridges to connect the deposited metal layer and oxidized graphene, and more covalent bridges are formed as graphene is more oxidized. However, the oxidation would etch graphene and oxidize the exposed copper substrate when the oxidation is beyond $7.7 \%$, resulting in a decrease in thermal conductance.

A common phenomenon existing in constrained 2D atomic layer interfaces is that the presence of graphene layer does not cause too much increase in thermal resistance. Instead, it can even reduce the overall resistance between metallic/non-metallic interfaces [25]. Sandwiched structure of graphene interface is different from the case of bare graphene on substrate. There are several influences from the coating of additional layer. First, the coating makes the graphene-substrate interface very tight and eliminates ripples. During sputtering coating, the atoms of coating material are pumped with a high speed and momentum, pushing graphene to substrate. The surface morphology of graphene could be significantly changed. During heating experiment, graphene is constrained between coating layer and substrate. Not much structural change could happen. Second, since graphene is only atomically thin, the bonding force between coating material and substrate still exists and the lattice vibration of the coating layer (adjacent to graphene) somehow introduces direct phonon transmission to substrate (tunneling effect). Thus, there should be direct energy exchange/coupling between them in addition to the transport through the interface, which somehow increases the apparent interface conductance. 
In TDTR experiment, the absorbed laser energy in the metallic layer is carried by electrons. As shown in Figure 3, the heat is transferred down to the interface through different mechanisms: absorbed photon energy is first transferred to the electrons. The energy is coupled through electron scattering and electron-phonon energy exchange in the metal layer. The mechanism of energy transport across graphene interfaces can be diverse. For graphene/semiconductor interfaces, phonon is the main energy carrier in both graphene and substrate. Therefore, the energy transport is mainly dominated by phonon transmission. At the metal layer/graphene interface, there are boundary scattering, phonon-phonon energy coupling, and electron-phonon energy exchange between the metal layer and graphene. At the graphene-substrate interface, there are again boundary scattering and the phonon-phonon energy coupling. Besides, there might be direct phonon energy exchange between coated layer and substrate material. The direct electron-phonon couplings could reduce the interfacial thermal resistance, whereas the indirect electron-phonon couplings between electrons in metal and phonons in non-metal through the near interface electron-phonon interactions on the metal side could increase thermal resistance. This is because the thermal energy needs to be transferred from phonons in the non-metal to the phonons in the metal, then from phonons in the metal to electrons in the metal, which forms series of thermal resistances. Therefore, the overall electron thermal transport between metal/non-metal interfaces depends on which channel dominates. It is noticed that the portion of electron scattering and electron/phonon coupling in total energy coupling is small in graphene interfaces [39]. The phonon-phonon coupling is the major energy exchange pathway for graphene interfaces. Koh et al [56]. found that phonon/phonon interaction still dominates the thermal transport across graphene/metal interfaces at temperatures 50-500 K. Majumdar et al [66]. concluded that the electron/phonon resistance only contributes to interfacial thermal transport when the phonon-mediated conductance is in the order of $\mathrm{GW} / \mathrm{K} \cdot \mathrm{m}^{2}$. Lyeo et al. [67] experimentally determined that electron scattering does not affect thermal transport 
across metal/diamond interfaces. In the results reviewed above, the graphene layer is sandwiched between two materials. The top layer sputtering could damage the graphene structure in addition to the ripple/wrinkle removal. However, this type of graphene structure damage/change is very difficult to characterize and is rarely characterized.

\subsection{Unconstrained 2D atomic-layer interfacial thermal transport}

Current work on direct thermal characterization of bare graphene interfaces are all based on Raman technique. The advantage is that it provides a direct measurement and the result is not affected by coating materials. Therefore, the measurement results reflect the intrinsic graphene interface energy transport. This knowledge is critical in terms of assessing the sample preparation process, and evaluating the interface bonding quality. Unfixed graphene interface could be relatively loose, and the interface exhibits larger thermal resistance than constrained ones. By using the electrical-heating Raman-probing method, Yue et al. characterized interfacial thermal resistance between tri-layer graphene and $\mathrm{SiC}$ as $5.3 \times 10^{-5} \mathrm{~K} \cdot \mathrm{m}^{2} / \mathrm{W}$ [14]. In the measurement, the thermal expansion induced interface mismatch is an important factor responsible for the large thermal contact resistance [14]. Chen et al. applied this method on interfacial thermal conductance measurement across graphene/h-BN and obtained a value as $7.41 \times 10^{6} \mathrm{~W} / \mathrm{m}^{2} \cdot \mathrm{K}$ [68]. The high electrical power applied on graphene could be the reason for this small value [68].

Joule heating effect can be partially solved by using another laser to heat a regional area only. Based on the optical heating method, Cai et al. characterized the interfacial thermal conductance between graphene and Si substrate as $28_{-9.2}^{+16} \mathrm{MW} / \mathrm{m}^{2} \cdot \mathrm{K}$ [61]. Judek et al. obtained the value of interfacial thermal conductance as $1.7 \mathrm{MW} / \mathrm{m}^{2} \cdot \mathrm{K}$ for graphene $/ \mathrm{SiO}_{2} / \mathrm{Si}[69]$. Tang et al. determined the thermal conductance $\left(G_{t}\right)$ as $183 \pm 10$ and $266 \pm 10 \mathrm{~W} / \mathrm{m}^{2} \cdot \mathrm{K}$ for graphene/Si and graphene $/ \mathrm{SiO}_{2}$ interfaces, which is five orders of magnitude lower than the normal thermal interfacial conductance [26]. Recent work 
also by Tang et al. reported a thermal conductance of $410 \pm 7 \mathrm{~W} / \mathrm{m}^{2} \cdot \mathrm{K}$ for graphene $/ \mathrm{SiC}$ interface [70]. The extremely low thermal conductance stems from the decoupling effect of phonon transport across the graphene interface due to the loose interface mechanical coupling, which is validated by strain analysis from Raman peak shift and interface optical interference. If a 2D material is sandwiched between two materials, it will be very difficult to assess the interface quality using a different technique, and use the result to explain the measured thermal conductance/resistance. For bare 2D material interface, the Raman intensity is strongly affected by any tiny local interface spacing (in the order of $\mathrm{nm})$. Therefore, the interface-induced optical interference can be used to assess the local interface quality. This provides a good way to interpret the measured interface thermal conductance/resistance [71].

\subsection{Interface structure revealed by Raman spectrum analysis}

During heating experiment, the strain effect induced by thermal stress is important and could lead to additional peak shift beside sole temperature effect [72]. Therefore, strictly speaking, temperature determination by sole peak shift is not very accurate for heating experiment with a large temperature range. The linewidth (full width at half maximum, FWHM) is only temperature dependent. Although its sensitivity is less than that of wave number $[72,73]$, it is still a good temperature indicator if the optical arrangement is carefully adjusted. Raman peak intensity is temperature sensitive and can be used for temperature probing because temperature not only influences the excited photon energy but also impacts the excitation efficiency [19]. However, thermal expansion effect during heating experiment is significant and could alter the optical path to a certain level. Nevertheless, Raman intensity can be used for probing interface structure, or validating corrugation problem in graphene interface, which is a common phenomenon encountered during the 2D material transfer to substrate. 
Interface delamination might induce light interference which could enhance Raman intensity. When an interface spacing exists, even at the level of $\mathrm{nm}$, it will induce multiple reflections of the excitation laser and Raman signal. The Raman signal will be either enhanced or reduced, depending on the interface spacing. In Tang et al.'s work, the Raman intensity was, for the first time, employed to study the interference effect between graphene and substrate to probe the delamination phenomenon at the interface $[26,70]$. In calibration experiment, the 2D material will have the same temperature as the substrate. In laser-heating experiment, the $2 \mathrm{D}$ material has a higher temperature than the substrate. The two scenario comparison will uncover the $2 \mathrm{D}$ material behavior and the change of the interface spacing. Detailed treatment and analysis can be found in the work by Tang et al $[26,70]$. Figure 4(a) shows schematic of this light enhancement theory induced by the corrugation of graphene layer on substrate. In Tang et al.'s work, absorbed energy flux in graphene is $3.8 \times 10^{4} \mathrm{~W} / \mathrm{m}^{2}$, and the temperature rise is obtained as $\Delta T=113.1 \mathrm{~K}$, and $\chi_{I}=-0.00131 \mathrm{~K}^{-1}$. The measured normalized Raman intensity is $I_{\exp } / I_{0}=0.890$. The expected normalized Raman intensity without interface separation change is $\left(1+\Delta T \cdot \chi_{I}\right)=0.852$. Therefore, the enhancement factor is $F_{\text {nor }}=1.045$. Based on AFM images, the original separation between graphene and substrate is roughly $\delta_{\text {cal }}=2.1 \mathrm{~nm}$, the original enhancement would be $F_{\mathrm{o}}=1.015$. After extra intensity enhancement by $\delta_{\exp } \rightarrow \delta_{\text {cal }}$, the final enhancement factor is $F_{h}=F_{o} \cdot F_{\text {nor }}=1.061 . F_{\mathrm{o}}$ and $F_{\mathrm{h}}$ are illustrated in Figure 4(b). Based on the enhancement calculation shown in Figure 4(c), the final separation distance will be $\delta_{\text {exp }}=5.0 \mathrm{~nm}$ for both parallel- and perpendicular-beam cases. The separation increment during laser heating is obtained as $\Delta \delta=\delta_{\text {exp }}-\delta_{\text {cal }}=2.9 \mathrm{~nm}[26$, 70].

The other evidence of interface separation is from measuring stress level during heating experiment. It is understood that interfacial materials experience different thermal expansion and thermal stress of materials derived from Raman spectrum can uncover 
how tight the interface is. For a loose interface, the strain of the 2D material will be very small. The peak shift and the linewidth of Raman spectrum can be combined to study this effect: the peak shift method involves the effect of thermal strain while the linewidth does not, the thermal strain can be distinguished by comparing measured results from peak shift and linewidth. In Tang et al.'s work, it is calculated that very small strain effect is involved during heating experiment, which double confirms that there is a relatively loose interface between graphene and substrate. In addition, the weak interface coupling due to the delamination effect explains the measured extremely low value (around 410 $\mathrm{W} / \mathrm{m}^{2} \cdot \mathrm{K}$ ) of interfacial thermal conductance. In all, Raman thermometry cannot only be used for interfacial thermal characterization, but also regarded as an effective tool to explore the structural change during heating experiment. Tang et al.'s results proved that the slight increase in the interface spacing will significantly decrease the interfacial thermal conductance and Raman-based dual thermal probing method provides a pathway for comprehensive study of complex structures of graphene interfaces, especially with complex structural problems $[26,70]$.

\section{Molecular dynamics modeling of interface energy coupling}

Contrast to experiment characterization, molecular dynamics study of interface energy coupling experiences little restrictions on structure design, and has the capacity of studying a very large scope of materials and interface structures. However, inter-atomic potential between the adjacent materials has to be constructed based on literature. The reported interface thermal conductance/resistance can be strongly affected by this potential selection. This is a problem often questioned in assessing the reported simulation results. Also if the system's temperature is well below the Debye temperature, quantum correction needs to be counted to give a more meaningful temperature of the material next to the interface. This could significantly complicate the problem since the two adjacent materials could have different Debye temperatures. Quantum correction is 
not used so often in interface thermal conductance study, and the reported results are restricted to the classical MD regime, where all phonon energy quantum levels are filled with the same chance.

\subsection{Steady-state method: non-equilibrium molecular dynamics}

Non-equilibrium molecular dynamics (NEMD) simulation is a steady-state, non-equilibrium numerical approach widely used to characterize interfacial thermal resistance. A temperature gradient can be built in the hybrid system by applying two heat reservoirs at the opposite ends in the heat flow direction, which is also named the direct NEMD ( $d$-NEMD) method. Alternatively, a heat flux can be directly added to the system by adding/subtracting kinetic energies, i.e., the reverse NEMD ( $r$-NEMD) method. The temperature drop occurring at the interface of the contact area can be used to determine the interfacial thermal resistance.

The NEMD technique has been successfully applied to the study of thermal transport in 1D structures like nanotube [74-76]. It can be used for studying the interfacial thermal transport between CNTs, or between CNT and substrate. In reality, different contact styles coexist between CNTs. For example, it can be cross-contact, parallel-contact, or contact at random angles. It can be connected by different linkers or by different contact strengths. Different scenarios of these contact styles can significantly affect the interfacial thermal transport in one or two orders of magnitude. Comprehensive studies of these effects via experimental characterizations are very difficult, whereas the NEMD simulation method provides a convenient and fast approach to understand the underlying mechanism for interfacial thermal transport between CNTs.

For 2D materials like graphene, $h$-BN, silicene, phosphorene, $\mathrm{MoS}_{2}$ and $\mathrm{MoSe}_{2}$, the NEMD method for interfacial thermal resistance predictions should be used with caution. 
If a heat flux is directly imposed on the $2 \mathrm{D}$ material, the temperatures calculated from this region could be illusory and the temperature jump at the interface will be inaccurate. This is because the continuous atomic velocity scaling in the 2D layer during heating will significantly change the system's energy distributions, introducing more and unrealistic phonon scatterings, and the calculated local temperature does not reflect its true value. To avoid this controversial situation, the $2 \mathrm{D}$ material can be put in the middle of a sandwiched structure, where the heat reservoirs are placed on the two sides of the bulk matrix. After the system reaches thermal equilibrium, the temperature of the $2 \mathrm{D}$ material and its adjacent layers will be recorded and used for the thermal contact resistance calculations. For this modeling treatment, the materials on both sides of the 2D material could have long-range interaction and exchange energy directly without via the 2D material (if the 2D material is very thin). This will change the interface energy coupling scenario, just like 2D material sandwiched between two materials in frequency-domain and time-domain characterizations. Also the extra material on both sides of the $2 \mathrm{D}$ material will constrain the phonon movement of the $2 \mathrm{D}$ material, thereby leading to undesired phonon alterations.

For three-dimensional (3D) bulk materials containing tens of atomic layers, the NEMD method has been extensively used to calculate both the thermal conductivity and interfacial thermal resistance [28, 77, 78]. After thermal equilibrium calculations, the thermal conductivity of each material can be calculated by linear fitting of the temperature profile. For a harmonic system, the energy equipartition exists between the kinetic and potential energies, and between the modes. In the temperature gradient building process, kinetic energies are constantly added/subtracted to/from the heating/cooling areas for temperature controls. In this ultrafast energy exchange process, the kinetic and potential energies are in non-equilibrium state and phonon boundary scattering is intensive at the interface between the heating/unheating (or 
cooling/uncooling) regions. Therefore, the temperature drop is non-uniform in these regions and must be excluded from thermal conductivity calculations [28, 79].

\subsection{Transient method: numerical pump-probe}

Aside from the steady-state NEMD method, transient heating techniques in molecular dynamics can also be used in interfacial thermal transport studies. A transient MD simulation approach has been developed following the principles of the experimental pump-probe technique, which is an optical thermal characterization method that has been used extensively for thermal characterization of micro/nanofilms [42]. After thermal equilibrium calculations, the supported 2D membrane is exposed to an ultrafast thermal impulse, which will cause a temperature rise in the monolayer system. In the following thermal relaxation processes, the surface temperature of the sample will decrease due to the heat conduction to the substrate. Since the only energy dissipation channel in the heterostructure is through the heated monolayer to the substrate, correlations between the temperature difference and energy evolution can be used to calculate the interfacial thermal resistance without the awareness of specific heat. This technique has been used in our research to study the interfacial thermal transport across graphene- $h$-BN [80], graphene-phosphorene [81], graphene-stanene [82], silicene-silicon [83], graphene-silicon [84] and graphene-copper [85] interfaces. Compared to the NEMD method, the transient technique focuses on the dynamic response of the hybrid system and therefore can greatly reduce the computation time. It also eliminates the undesired phonon scattering in the 2D material due to continuous velocity scaling.

\subsection{Theoretical methods based on local phonon scattering}

Theoretical methods using the acoustic mismatch model (AMM) [86-88] and diffuse mismatch model (DMM) [89-92] are widely used to study interfacial thermal resistance properties at low temperatures. The AMM assumes that the interface between two 
materials is perfectly specular and the phonons either transmitting or reflecting at the interface should obey Snell's law. However, this ideal case only represents a limited number of modern devices. To better describe the interface phonon scattering, the diffuse mismatch model is developed. The DMM assumes that when crossing the interface, the phonons will lose track of which side of the interface they come from, as well as their former directions and polarizations. The transmissivity has no angular or phonon mode dependency because of the nature of diffusive scattering. Both AMM and DMM can predict experimental data quite well at low temperatures [92-94]. However, at high temperatures, most of the practical material systems deviate significantly from assumptions used in theoretical studies. This will cause great discrepancy in the interfacial thermal resistance between model predictions and experimental results. Recently, a frequency dependent form of the acoustic mismatch model referred to as the phonon mismatch model (PMM) is brought up to describe the interfacial thermal conductance in presence of an impurity mass between the interfaces [95, 96]. In general, materials with similar vibrational properties will have a higher transmissivity, while the converse is true for mismatched materials. By applying the PMM model, it is proved that the interface phonon transmission can be maximized for all frequencies by setting the interface mass equal to the arithmetic mean of the masses on either side of the interface. Another empirical formulation of phonon transmissivity termed thermal mismatch model (TMM) is developed to predict transmissivity at ideal interfaces between semiconductor materials $[97,98]$. The TMM has been successfully applied to identify ways to tune the transmissivity of multilayered structures and superlattices. It is shown that by introducing intermediate layers of certain atomic masses, the total transmissivity can either be systematically enhanced or reduced compared to that of a single interface. Based on the traditional DMM model, a revised mismatch theory is proposed assuming the phonon scatterings at the interfaces are contributed by two individual components [90]. The first part accounts for phonons that are scattered at the interface as described by the traditional 
mismatch theories. The second part accounts for phonons that do not see the interface but instead are scattered once they have propagated into the adjacent interface. At an atomically flat interface, it may not be physically reasonable to assume that all phonons will scatter as a result of the mismatch between the hetero materials. Instead, due to phonon wavelength and mean free path deviations, many phonons may not scatter until they transport a certain distance into the other material. This model is in good agreement with classical MD simulations of phonon thermal transport across $\mathrm{Si} / \mathrm{Ge}$ interfaces. A summary of simulation and theoretical calculation results is presented in Table 2.

\section{Interfacial thermal transport based on MD simulations}

Although thermal resistance/conductance based on MD simulations could not recover the true scenarios studied in experiment and cannot be compared with experimental results quantitatively, they do provide a great reference to compare with the experimental results and assess the interface quality. Also the simulation sheds light on the mechanisms of interface energy coupling, and provides guidance on interface energy transport control.

\subsection{Thermal contact resistance in zero-to-one dimensional structures}

As discussed in the characterization sections, the synthesis of 1D nanostructure contact in experiment is far more difficult than in modeling. Therefore, MD simulation, which can precisely control and generate perfect structure of CNT contact, can help us understand the mechanism and provide the guidelines on thermal design of 1D nanostructures. Maruyama et al [99]. characterized the thermal conductance of parallel contact of CNTs as $4.04 \mathrm{MW} / \mathrm{m}^{2} \mathrm{~K}$. Zhong et al [100]. modeled heat conduction through two aligned CNTs with one ends overlapping (as shown in Figure 5a) and the other ends setting as the hot and cold spots respectively. The thermal contact resistance is characterized as $1.1 \times 10^{-7} \mathrm{~K} \cdot \mathrm{m}^{2} / \mathrm{W}$ for $5 \mathrm{~nm}$ tubes with $2.5 \mathrm{~nm}$ overlap. Evans et al [101]. studied the inter-tube thermal conductance between CNTs as a function of crossing angle, pressure, 
and thermal property of bulk bundles. Varshney et al [102]. simulated the thermal conductance of CNTs contacted with organic linkers. They found that the interfacial conductance increases in a nonlinear relationship with the number of $\mathrm{CH}_{2}$ linkages. There are many other reported results for different contact modes of CNTs [103-106]. Thermal contact resistance between $\mathrm{CNT}$ and other materials such as $h$-BN [107], $\mathrm{Cu}$ nanowires [108]. Chen et al [109]. investigated the effect of chemical functionalization on interfacial thermal conductance between CNT/CNT by connecting the CNTs with different number of $\mathrm{CH}_{2}$ chemical linkers as shown in Figure 5(b). It is found that thermal conductance can be increased greatly and there is an optimized linker number for thermal transport enhancement.

For the study of thermal contact between CNT and substrate, Ong and Pop simulated thermal transport between $\mathrm{CNT}$ and $\mathrm{SiO}_{2}$ substrate [110]. Similar to their experimental work, the structure in MD simulations is a lateral CNT on substrate. It is found that thermal conductance is increased with increasing bonding strength between CNT and substrate, CNT diameter and temperature. $\mathrm{Hu}$ et al. studied thermal contact resistance between a standing CNT and Si substrate and found that existence of the chemical bond between $\mathrm{CNT}$ and Si can effectively improve the interfacial thermal transport (two orders of magnitude) [111]. Bao et al. investigated the interfacial thermal conductance between CNT and silicon in two situations: CNT is in contact with silicon directly and CNT is in contact with silicon through CNT-graphene junction [112]. They found that the interfacial thermal conductance between CNT/silicon interface with CNT-graphene junction is improved by $40 \%$ compared to that between pure CNT/silicon interface with covalent bonding, and the interfacial thermal conductance enhancement is almost one order of magnitude higher compared to van der Waals bonding. Feng et al. studied thermal conductance at the interface of single-wall CNT arrays and silicon [113]. Shi et al. built a 3D CNT-graphene network model as shown in Figure 5(c) to investigate the 
thermal transport in the network structure and found that the overall thermal resistance primarily comes from CNT-graphene junctions [114]. Ni et al. reported the values of thermal resistance for CNT-HLK5/polystyrene, CNT-HLK5/epoxy and CNT-HLK5/polyethylene contacts are $7 \times 10^{-9}, 6.6 \times 10^{-9}$ and $6.6 \times 10^{-9} \mathrm{~K} \cdot \mathrm{m}^{2} / \mathrm{W}$, respectively for the CNT/polymer matrix interface, and found that $\mathrm{HLK} 5\left(\mathrm{C}_{22} \mathrm{H}_{25} \mathrm{O}_{3} \mathrm{~N}_{3}\right)$ functionalization can efficiently improve thermal transport [115]. In summary, the simulations of thermal contact resistance have been published far more than experimental work, partly demonstrating the great difficulty faced in experimental work: sample preparation and characterization. The drawback of MD simulations is the results are highly dependent on interatomic potentials.

\subsection{Effects of surface roughness}

Aside from bent structures in nanodevices, the substrate surfaces are often dented with patterns to achieve maximum thermal radiation and realize various electrical functions. Therefore, the effect of surface roughness on the thermal transport across graphene-substrate is of great interest for exploration. Details of the hybrid graphene/copper systems modeling can be found in our past work [85]. It is very surprising and interesting to observe that the interfacial thermal resistance first decreases as groove depth $(\delta)$ becomes larger. This is contrary to the traditional thought that, in comparison with a flat surface, a rough surface should always give a larger interfacial thermal resistance due to the poor contact.

To explain these new findings, the interatomic forces between graphene and copper are calculated for the $\delta=6.3 \AA$ case and the results are shown in Figure 6. Due to the roughness of the copper surface, the interatomic forces are not evenly distributed in the supported graphene. For graphene over the nanogroove, most of the $\mathrm{C}$ - $\mathrm{Cu}$ distance is large, beyond the repulsive force range. The $\mathrm{C}-\mathrm{Cu}$ interaction is attractive. When the 
nanogroove depth is small, this attractive force is strong enough to bend the graphene to fit the copper surface. Since the overall force on the graphene is zero on average, a net repulsive force will arise for the supported graphene areas. This is like the supported graphene region is pulled down on both sides by the attractive force in the suspended regions. The significant enhancement of the local contact pressure in the supported graphene region leads to a decreased thermal resistance between graphene and copper. This thermal resistance decrease offsets the thermal resistance increase in the suspended region, giving an overall thermal resistance decrease.

\subsection{Effects of substrate morphology}

Phonon transmission through graphene interfaces is affected not only by the atomic interactions but also by the substrate structure [116-120]. One recent work investigated the interfacial thermal transport across graphene/crystalline $\mathrm{SiC}(c-\mathrm{SiC})$ and graphene/amorphous $\mathrm{SiC}(a-\mathrm{SiC})$ interfaces [28]. The resistance between graphene and $a$-SiC is computed as $3.49 \pm 0.08 \times 10^{7} \mathrm{~W} / \mathrm{m}^{2} \mathrm{~K}$, which is larger than both graphene/C-terminated $c$-SiC and graphene/Si-terminated $c$-SiC interfaces. The abnormal phenomenon can be explained by the difference in vibrational properties of crystalline solid and amorphous solid. Phonon density of states (PDOS) is calculated for understanding this phenomenon. The phonon PDOS of $a-\mathrm{SiC}$ is much smoother than the phonon PDOS of either adjacent $\mathrm{C}$ atoms or $\mathrm{Si}$ atoms under van der Waals interaction. The wide phonon PDOS allows larger possibility for phonon transmission across the interface and improves the overlap area of the phonon PDOS of graphene and that of $a$-SiC. Besides, the rough surface feature of amorphous solids may be partly responsible for the improved interfacial thermal conductance, which has been verified by Zhang et al [84]. that sub-nanoscale roughness might be good for interfacial energy coupling. The fact that the interfacial thermal conductance of graphene/a-SiC is larger than that of graphene $/ c-\mathrm{SiC}$ gives us a new interpretation of the thermal transport in amorphous 
structure. In traditional understanding, amorphous solids transport heat far slower than its crystalline counterpart due to the short phonon mean free path. However, when it comes to the interfacial thermal transport where the intrinsic phonon mean free path of one component at the interface does not dominate the thermal conductance, the situation becomes different.

This conclusion is further confirmed by another recent interfacial thermal transport study between silicene and $c-\mathrm{Si}, c-\mathrm{SiO}_{2}, a-\mathrm{Si}$ and $a-\mathrm{SiO}_{2}$ [83]. The higher interfacial thermal conductance at a disordered interface was also observed by English et al [98]. The effect of different levels of disorder on the interfacial thermal conductance was investigated and the medium level of disorder was proven to be most beneficial to the interfacial thermal transport.

\subsection{Effects of chemical functionalization}

Functionalization of 2D material through chemical methods has attracted great interest and has been explored recently due to the possibility of nanostructuring $2 \mathrm{D}$ material into complex patterns [121-123]. It has been proved that thermal transport in graphene is highly anisotropic. Thermal energies in graphene are mainly carried by the out-of-plane flexural phonons (ZA) whereas the in-plane transverse (TA) and longitudinal phonons (LA) play less significant roles. One the other hand, the energy couplings between $\mathrm{ZA} \leftrightarrow \mathrm{TA} / \mathrm{LA}$ phonons are much slower than those between TA $\leftrightarrow$ LA. Therefore, aside from the thermal resistance across the boundary, another bottleneck in interfacial thermal transport is the energy transfer rate between ZA and TA/LA phonons in the monolayer.

The doped atoms at graphene surface broke the $s p^{2}$ structures between C-C bonds and act as scattering centers for $\mathrm{C}$ atoms, which will facilitate the energy transfers among different phonon modes. As a result, chemical functionalization has been proved to be an 
effective way to manipulate the anisotropic thermal transport in graphene as well as its interfacial thermal transport properties.

Thermal properties of hydrogenated graphene (H-GNR) and oxidized graphene (O-GNR) were investigated by several studies to seek better thermal management and thermoelectric applications [124-126]. Interfacial thermal conductance $(G)$ across stacked GNR and silicene can be tuned by adjusting the hydrogen coverage $(f)$ on graphene. When hydrogen atoms are randomly distributed on both sides of the GNR, $G$ has a non-monotonic trend with $f$. With hydrogen coverage of $50 \%$, thermal conductance between GNR and silicene can be increased to a maximum value of $61.75 \mathrm{MW} / \mathrm{m}^{2} \cdot \mathrm{K}$ [127]. The physical mechanism can be explained by phonon power spectrum analyses on the hybrid structure. Enhanced phonon couplings between $\mathrm{C}-\mathrm{Si}$ atoms and improved intra-modes phonon couplings within GNR are the main reasons for the thermal conductance increase. Compared with pristine graphene, it has been proved that the interfacial thermal resistance between H-GNR and hexagonal boron nitride $(h$-BN) can be reduced by $76.3 \%$ [80]. Since the in-plane and out-of-plane phonons in graphene are highly decoupled, the thermal energies are restrained in lateral directions and can only be slowly released to the flexural phonons. By hydrogenating graphene in the heterostructure, the coupling between in-plane and out-of-plane phonons can be greatly enhanced. As the hydrogenation ratio increases, the overlap area between in-plane and out-of-plane phonons in H-GNR greatly increases. The improvement of in-plane and out-of-plane phonon couplings in graphene indirectly facilitates the thermal transport across interface and reduces the thermal contact resistance. In the meanwhile, the overlap area between H-GNR and $h$-BN's out-of-plane direction phonons also increases with higher hydrogenation ratio, which directly contributes to the thermal conductance across the interface. 


\section{Challenges and opportunities in energy coupling at atomic layer interfaces}

\subsection{Challenges confronted in current thermal characterization techniques}

The surface coating on 2D material could modify contacting mode, leading to a much tighter interface which reduces interfacial thermal resistance. In addition, there are not many scenarios in real applications that require the 2D material coated since this would significantly affect its performance. Much attention should be paid on the accurate measurement of thermal contact resistance of bare graphene interface structures. The direct temperature measurement of both sides of the interface especially at the nanoscale is a great challenge for any traditional optical/electrical thermometry. Only Raman thermometry which is based on the scattering photon energy of the probe light rather than the light intensity can be effective to define temperature differential in such a small scale. The important concerns in Raman thermometry are additional heating effect from probing laser and limitation in temperature probing uncertainty.

Usually, the high accuracy of Raman measurement requires high-end resolution in Raman spectrum for determining temperature. Meanwhile, a sound Raman signal is preferred for either peak fitting or intensity determination. However, sound Raman signal requires strong laser intensity. Not significant but still non-negligible absorption from Raman probing laser results in additional heating besides applied heating current or heating laser. This undesired heating also induces thermal stress or even expansion in $2 \mathrm{D}$ material which induces inaccuracy in thermal characterization. Strong enough Raman signal without powerful heating is a tradeoff for using Raman thermometry in bare 2D material interface characterization. One alternative is to develop a new Raman technique to avoid intensive laser heating and realize accurate temperature control. The transient Raman technique which is based on a pulsed laser working as the heating source and probing light might be a good choice, which is being attempted in Wang's lab [128]. Precedent work includes the successful measurement of thermal diffusivity of silicon 
cantilever [128, 129]. and CNT fiber [130].

\subsection{Manipulation of interfacial thermal transport at the atomic scale}

Many conditions cannot be ignored considering the complex scenario at the $2 \mathrm{D}$ material interface. For example, the functional groups and other chemical residues on the 2D material surface during synthesis make the material not just single layer of atoms. The functional group would definitely interact with substrate material and thus could improve or alleviate the interfacial thermal transport. For CNT's contact, the main barrier is from the limited contact area as analyzed in above sections. Improving the interaction between the tubes is the most effective way to reduce thermal contact resistance. Kaur et al. reported that the interfacial thermal resistance between metal and vertically aligned multiwall CNT arrays can be greatly reduced by bridging the interface with covalently bonded organic molecules [131]. Therefore, functionalization of 1D nanostructures is the key for a successful thermal bonding.

In addition, the temperature induced thermal stress might result in interface delamination or corrugation problems. In such cases, the interfacial thermal transport is greatly affected and the thermal conductance might be reduced in orders of magnitude. For a loose contact with van der Waals force, the thermal conductance of which can be one or two orders of magnitude smaller than that of covalent bond, might be better for releasing thermal stress and thus preventing interface delamination and corrugation problems. For bare 2D material interfaces which already have corrugation or wrinkling problems, a clearer understanding of phonon transmission mechanism through such weakly coupled interfaces is important. The dual temperature probing method combined with interface structural analysis from Raman intensity as mentioned in Tang et al.'s work might be the way to tackle this problem $[26,70]$. 


\subsection{Other 2-D materials beyond graphene}

Other 2-D materials beyond graphene, such as silicone [132], hexagonal boron nitride ( $h$-BN) [133] and $\mathrm{MoS}_{2}$ [134] might also possess extraordinary properties since the monolayer structure is similar while might even better since the zero band gap of graphene impedes its abroad applications in transistors. $\mathrm{MoS}_{2}$ is an atomic-layer material with a small band gap which can be useful in nanoelectronics. The thermal conductivity of few-layer $\mathrm{MoS}_{2}$ has been measured by Raman thermometry as around $52 \mathrm{~W} / \mathrm{m} \cdot \mathrm{K}$ [134]. For silicene, no experimental data on the thermal conductivity has been reported yet since the synthesis of large scale samples is still a challenge. Recently black phosphorus has been discovered to possess extraordinary electronic properties and the black phosphorus based transistor has been reported [135]. Tons of opportunities exist in the study of thermal transport across these atomic structured materials.

Meanwhile, thermal transport in their interfacial structures might be an interesting problem to study. Besides, $\mathrm{MoS}_{2}$ has strong Raman excitation [136, 137], thus the Raman thermometry should be well applied in its interface thermal characterization. Recently, Taube $e t$ al. used Raman thermometry to characterize the interfacial thermal conductance of $\mathrm{MoS}_{2}$ on $\mathrm{SiO}_{2}$ as $1.94 \mathrm{MW} / \mathrm{m}^{2} \cdot \mathrm{K}$ and $1.25 \mathrm{MW} / \mathrm{m}^{2} \cdot \mathrm{K}$ at temperatures $300 \mathrm{~K}$ and $450 \mathrm{~K}$ respectively [138]. Thermal properties of other 2D materials, such as silicene, $h$-BN have been studied as well. The interfacial thermal transport between silicene and various substrate has been studied by MD simulations [83].

\subsection{Bulk material involving interfacial thermal transport of atomic-layer structures}

The scale-up of low-dimensional materials towards industrial applications is the ultimate goal of material research. Many bulk materials based on CNT and graphene, e.g., CNT fibers [139], buckypaper [20], graphene foam [140-142], also attracted tons of interests with more and more characterization results are being reported. The reduced thermal 
property of bulk materials is attributed to the numerous thermal contacts and the alignment between them [143]. Pettes et al. reported that the thermal conductivity of graphene foam decreases with temperature above room temperature. It is due to the Umklapp phonon scattering effect inside graphene flakes [141]. However, Li et al. found that a different kind of graphene foam behaves differently [140]. The thermal conductivity is increased with increasing temperature similarly to other porous materials. It is regarded that the thermal contact resistance between graphene flakes rather than graphene itself dominates thermal transport of bulk graphene foam samples [140]. Zeng et al. backfilled PMMA into the pores of graphene aerogel (GA) to obtain GA-PMMA composites and found that thermal conductivity of composites is increased with volume fraction of graphene [144]. A clearer understanding and more comprehensive description of energy coupling between atomic layer materials is essential for thermal design of bulk materials. Besides, alignment is equally important for bulk materials. The inner structure can greatly change the effective thermal conductivity of materials. However, considering large systems of inner structures, the accurate prediction of thermal property of bulk materials is still a challenge [143].

\section{Concluding Remarks}

Understanding energy coupling across atomic-layer interfaces is essential to the implementation of these structures into energy-based applications or thermal design of sensors and electronic devices. To experimentally characterize the thermal contact resistance, steady state techniques, frequency domain techniques, and time domain techniques have been used. For 1D nanostructures, the synthesis of the contact is a great challenge. Also the final determination of the contact thermal resistance needs knowledge of thermal conductivity of the 1D nanostructure. For 2D atomic layer-substrate interfaces, extra material preparation (e.g. coating on the 2D material) will make many techniques applicable, but also introduce extra problems in the material structure and interface 
energy coupling. Raman-based techniques can be used to characterize free $2 \mathrm{D}$ material-substrate interfaces. The corrugation and weak coupled interface is responsible for the reported low thermal conductance. However, precise determination of the laser absorption of the 2D material is a key factor in determining the measurement accuracy, and should be treated with great measures. Contrast to experimental work, MD simulations experience much less structure constrain and have reported much more interface energy coupling studies. Temperature quantum correction is rarely used, and the reported interface thermal conductance/resistance can only be compared with experimental work qualitatively. Nevertheless, MD simulations provide great understanding of the mechanisms behind interface energy transport. More defined heating, temperature measurement improvement, and more in-depth structure understanding are three great research directions toward better understanding of interface energy coupling of atomic-scale materials. To improve the contact energy coupling, the local structure can be manipulated physically and chemically to enhance phonon/electron energy exchange.

\section{Acknowledgements}

The financial support from National Natural Science Foundation of China (Nos. 51428603 and 51576145), National Science Foundation (CBET1235852, CMMI1264399), Department of Energy (DENE0000671, DE-EE0007686), and Iowa Energy Center (MG-16-025, OG-17-005) is gratefully acknowledged. 


\section{Reference}

[1] S.J. Tans, A.R. Verschueren and C. Dekker, Room-temperature transistor based on a single carbon nanotube, Nature, 393(6680) (1998) 49-52

[2] A.K. Geim and K.S. Novoselov, The rise of graphene, Nature materials, 6(3) (2007) 183-191

[3] R.H. Baughman, A.A. Zakhidov and W.A. de Heer, Carbon nanotubes-the route toward applications, Science, 297(5582) (2002) 787-792

[4] A.A. Balandin, S. Ghosh, W. Bao, I. Calizo, D. Teweldebrhan, F. Miao and C.N. Lau, Superior Thermal Conductivity of Single-Layer Graphene, Nano Letters, 8(3) (2008) 902-907

[5] M. Biercuk, M.C. Llaguno, M. Radosavljevic, J. Hyun, A.T. Johnson and J.E. Fischer, Carbon nanotube composites for thermal management, Applied Physics Letters, 80(15) (2002) 2767-2769

[6] S. Ghosh, I. Calizo, D. Teweldebrhan, E. Pokatilov, D. Nika, A. Balandin, W. Bao, F. Miao and C. Lau, Extremely high thermal conductivity of graphene: Prospects for thermal management applications in nanoelectronic circuits, Applied Physics Letters, 92(15) (2008) 151911

[7] L.A. Jauregui, Y. Yue, A.N. Sidorov, J. Hu, Q. Yu, G. Lopez, R. Jalilian, D.K. Benjamin, D.A. Delkd and $\mathrm{W}$. Wu, Thermal transport in graphene nanostructures: Experiments and simulations, Ecs Transactions, 28(5) (2010) 73-83

[8] L.B. Kish, End of Moore's law: thermal (noise) death of integration in micro and nano electronics, Physics Letters A, 305(3) (2002) 144-149

[9] E. Pop, Energy dissipation and transport in nanoscale devices, Nano Research, 3(3) (2010) 147-169

[10] S. Chen, A.L. Moore, W. Cai, J.W. Suk, J. An, C. Mishra, C. Amos, C.W. Magnuson, J. Kang and L. Shi, Raman measurements of thermal transport in suspended monolayer graphene of variable sizes in vacuum and gaseous environments, ACS Nano, 5(1) (2010) 321-328

[11] J.-U. Lee, D. Yoon, H. Kim, S.W. Lee and H. Cheong, Thermal conductivity of suspended pristine graphene measured by Raman spectroscopy, Physical Review B, 83(8) (2011) 081419

[12] S. Chen, Q. Wu, C. Mishra, J. Kang, H. Zhang, K. Cho, W. Cai, A.A. Balandin and R.S. Ruoff, Thermal conductivity of isotopically modified graphene, Nature materials, 11(3) (2012) 203-207

[13] J.H. Seol, I. Jo, A.L. Moore, L. Lindsay, Z.H. Aitken, M.T. Pettes, X. Li, Z. Yao, R. Huang, D. Broido, N. Mingo, R.S. Ruoff and L. Shi, Two-Dimensional Phonon Transport in Supported Graphene, Science, 328(5975) (2010) 213-216

[14] Y. Yue, J. Zhang and X. Wang, Micro/Nanoscale Spatial Resolution Temperature Probing for the Interfacial Thermal Characterization of Epitaxial Graphene on 4H - SiC, Small, 7(23) (2011) 3324-3333

[15] M. Fujii, X. Zhang, H. Xie, H. Ago, K. Takahashi, T. Ikuta, H. Abe and T. Shimizu, Measuring the thermal conductivity of a single carbon nanotube, Physical Review Letters, 95(6) (2005) 065502

[16] S. Berber, Y.-K. Kwon and D. Tománek, Unusually high thermal conductivity of carbon nanotubes, Physical Review Letters, 84(20) (2000) 4613

[17] P. Kim, L. Shi, A. Majumdar and P. McEuen, Thermal transport measurements of individual multiwalled nanotubes, Physical Review Letters, 87(21) (2001) 215502

[18] Y. Chalopin, S. Volz and N. Mingo, Upper bound to the thermal conductivity of carbon nanotube 
pellets, Journal of Applied Physics, 105(8) (2009) 084301

[19] Y. Yue, G. Eres, X. Wang and L. Guo, Characterization of thermal transport in micro/nanoscale wires by steady-state electro-Raman-thermal technique, Applied Physics A, 97(1) (2009) 19-23

[20] Y. Yue, X. Huang and X. Wang, Thermal transport in multiwall carbon nanotube buckypapers, Physics Letters A, 374(40) (2010) 4144-4151

[21] J. Yang, S. Waltermire, Y. Chen, A.A. Zinn, T.T. Xu and D. Li, Contact thermal resistance between individual multiwall carbon nanotubes, Applied Physics Letters, 96(2) (2010) 023109

[22] J. Yang, M. Shen, Y. Yang, W.J. Evans, Z. Wei, W. Chen, A.A. Zinn, Y. Chen, R. Prasher, T.T.

$\mathrm{Xu}, \mathrm{P}$. Keblinski and D. Li, Phonon Transport through Point Contacts between Graphitic Nanomaterials, Physical Review Letters, 112(20) (2014) 205901

[23] Y. Yue, J. Zhang, X. Tang, S. Xu and X. Wang, Thermal transport across atomic-layer material interfaces, Nanotechnology Reviews, (2015)

[24] Z. Chen, W. Jang, W. Bao, C.N. Lau and C. Dames, Thermal contact resistance between graphene and silicon dioxide, Applied Physics Letters, 95(16) (2009)

[25] C.W. Zhang, W.W. Zhao, K.D. Bi, J. Ma, J.L. Wang, Z.H. Ni, Z.H. Ni and Y.F. Chen, Heat conduction across metal and nonmetal interface containing imbedded graphene layers, Carbon, 64((2013) 61-66

[26] X. Tang, S. Xu, J. Zhang and X. Wang, Five Orders of Magnitude Reduction in Energy Coupling across Corrugated Graphene/Substrate Interfaces, ACS Applied Materials \& Interfaces, (2014)

[27] A. Ismach, C. Druzgalski, S. Penwell, A. Schwartzberg, M. Zheng, A. Javey, J. Bokor and Y. Zhang, Direct chemical vapor deposition of graphene on dielectric surfaces, Nano Letters, 10(5) (2010) $1542-1548$

[28] M. Li, J. Zhang, X. Hu and Y. Yue, Thermal transport across graphene/SiC interface: effects of atomic bond and crystallinity of substrate, Applied Physics A, 119(2) (2015) 415-424

[29] C. Yu, L. Shi, Z. Yao, D. Li and A. Majumdar, Thermal Conductance and Thermopower of an Individual Single-Wall Carbon Nanotube, Nano Letters, 5(9) (2005) 1842-1846

[30] L. Shi, D. Li, C. Yu, W. Jang, D. Kim, Z. Yao, P. Kim and A. Majumdar, Measuring Thermal and Thermoelectric Properties of One-Dimensional Nanostructures Using a Microfabricated Device, Journal of Heat Transfer, 125(5) (2003) 881-888

[31] N. Velson and X. Wang, Characterization of thermal transport across single-point contact between micro-wires, Applied Physics A, 110(2) (2013) 403-412

[32] J. Guo, X. Wang and T. Wang, Thermal characterization of microscale conductive and nonconductive wires using transient electrothermal technique, Journal of Applied Physics, 101(6) (2007) 063537

[33] E. Pop, D.A. Mann, K.E. Goodson and H.J. Dai, Electrical and thermal transport in metallic single-wall carbon nanotubes on insulating substrates, Journal of Applied Physics, 101(9) (2007)

[34] E. Pop, The role of electrical and thermal contact resistance for Joule breakdown of single-wall carbon nanotubes, Nanotechnology, 19(29) (2008) 295202

[35] L. Shi, J. Zhou, P. Kim, A. Bachtold, A. Majumdar and P.L. McEuen, Thermal probing of energy dissipation in current-carrying carbon nanotubes, Journal of Applied Physics, 105(10) (2009) 104306

[36] B.A. Cola, J. Xu, C. Cheng, X. Xu, T.S. Fisher and H. Hu, Photoacoustic characterization of 
carbon nanotube array thermal interfaces, Journal of Applied Physics, 101(5) (2007) 054313

[37] L. Guo, J. Wang, Z. Lin, S. Gacek and X. Wang, Anisotropic thermal transport in highly ordered TiO2 nanotube arrays, Journal of Applied Physics, 106(12) (2009) 123526

[38] Y. Yue and X. Wang, Nanoscale thermal probing, Nano reviews, 3((2012)

[39] P.E. Hopkins, M. Baraket, E.V. Barnat, T.E. Beechem, S.P. Kearney, J.C. Duda, J.T. Robinson and S.G. Walton, Manipulating Thermal Conductance at Metal-Graphene Contacts via Chemical Functionalization, Nano Letters, 12((2012) 590-595

[40] C. Riedl, U. Starke, J. Bernhardt, M. Franke and K. Heinz, Structural properties of the graphene- $\mathrm{SiC}(0001)$ interface as a key for the preparation of homogeneous large-terrace graphene surfaces, Physical Review B, 76(24) (2007) 245406

[41] X. Wang, H. Hu and X. Xu, Photo-acoustic measurement of thermal conductivity of thin films and bulk materials, Journal of Heat Transfer, 123(1) (2001) 138-144

[42] D.G. Cahill, K. Goodson and A. Majumdar, Thermometry and thermal transport in micro/nanoscale solid-state devices and structures, Journal of Heat Transfer, 124(2) (2002) 223-241

[43] R.T. Swimm, Photoacoustic determination of thin - film thermal properties, Applied Physics Letters, 42(11) (1983) 955-957

[44] J. Alexandre, F. Saboya, B. Marques, M.L. Ribeiro, C. Salles, M. Da Silva, M. Sthel, L. Auler and H. Vargas, Photoacoustic thermal characterization of kaolinite clays, Analyst, 124(8) (1999) 1209-1214

[45] A.J. Schmidt, R. Cheaito and M. Chiesa, A frequency-domain thermoreflectance method for the characterization of thermal properties, Rev Sci Instrum, 80(9) (2009) 094901

[46] J. Yang, E. Ziade, C. Maragliano, R. Crowder, X. Wang, M. Stefancich, M. Chiesa, A.K. Swan and A.J. Schmidt, Thermal conductance imaging of graphene contacts, Journal of Applied Physics, 116(2) (2014) 023515

[47] S. Majumdar, J.A. Sierra-Suarez, S.N. Schiffres, W.L. Ong, C.F. Higgs, 3rd, A.J. McGaughey and J.A. Malen, Vibrational mismatch of metal leads controls thermal conductance of self-assembled monolayer junctions, Nano Lett, 15(5) (2015) 2985-91

[48] M. Jeong, J.P. Freedman, H.J. Liang, C.-M. Chow, V.M. Sokalski, J.A. Bain and J.A. Malen, Enhancement of Thermal Conductance at Metal-Dielectric Interfaces Using Subnanometer Metal Adhesion Layers, Physical Review Applied, 5(1) (2016)

[49] D.G. Cahill, M. Katiyar and J.R. Abelson, Thermal conductivity of silicon thin films, Physical Review B, 50(9) (1994) 6077-6081

[50] T. Borca-Tasciuc, A. Kumar and G. Chen, Data reduction in $3 \omega$ method for thin-film thermal conductivity determination, Review of Scientific Instruments, 72(4) (2001) 2139-2147

[51] T. Tong and A. Majumdar, Reexamining the 3-omega technique for thin film thermal characterization, Review of Scientific Instruments, 77(10) (2006) -

[52] C.A. Paddock and G.L. Eesley, Transient thermoreflectance from thin metal films, Journal of Applied Physics, 60(1) (1986) 285-290

[53] R.M. Costescu, M.A. Wall and D.G. Cahill, Thermal conductance of epitaxial interfaces, Physical Review B, 67(5) (2003) 054302

[54] B.C. Gundrum, D.G. Cahill and R.S. Averback, Thermal conductance of metal-metal interfaces, 
Physical Review B, 72(24) (2005) 245426

[55] K.F. Mak, C.H. Lui and T.F. Heinz, Measurement of the thermal conductance of the graphene/SiO2 interface, Applied Physics Letters, 97(22) (2010)

[56] Y.K. Koh, M.-H. Bae, D.G. Cahill and E. Pop, Heat Conduction across Monolayer and Few-Layer Graphenes, Nano Letters, 10((2010) 4363-4368

[57] P.A. Vasquez Guzman, A. Sood, M.J. Mleczko, B. Wang, H.-S.P. Wong, Y. Nishi, M. Asheghi and K.E. Goodson, Cross plane thermal conductance of graphene-metal interfaces Orlando, FL (2014) 1385-1389

[58] I.-K. Hsu, R. Kumar, A. Bushmaker, S.B. Cronin, M.T. Pettes, L. Shi, T. Brintlinger, M.S. Fuhrer and J. Cumings, Optical measurement of thermal transport in suspended carbon nanotubes, Applied Physics Letters, 92(6) (2008) 063119-063119-3

[59] I. Calizo, A. Balandin, W. Bao, F. Miao and C. Lau, Temperature dependence of the Raman spectra of graphene and graphene multilayers, Nano Letters, 7(9) (2007) 2645-2649

[60] R. Nair, P. Blake, A. Grigorenko, K. Novoselov, T. Booth, T. Stauber, N. Peres and A. Geim, Fine structure constant defines visual transparency of graphene, Science, 320(5881) (2008) 1308-1308 [61] W. Cai, A.L. Moore, Y. Zhu, X. Li, S. Chen, L. Shi and R.S. Ruoff, Thermal Transport in Suspended and Supported Monolayer Graphene Grown by Chemical Vapor Deposition, Nano Letters, 10((2010) 1645-1651

[62] H. Maune, H.-Y. Chiu and M. Bockrathh, Thermal resistance of the nanoscale constrictions between carbon nanotubes and solid substrates, Applied Physics Letters, 89((2006) 013109

[63] B.M. Foley, S.C. Hernandez, J.C. Duda, J.T. Robinson, S.G. Walton and P.E. Hopkins, Modifying Surface Energy of Graphene via Plasma-Based Chemical Functionalization to Tune Thermal and Electrical Transport at Metal Interfaces, Nano Lett, 15(8) (2015) 4876-82

[64] Y.K. Koh, A.S. Lyons, M.H. Bae, B. Huang, V.E. Dorgan, D.G. Cahill and E. Pop, Role of Remote Interfacial Phonon (RIP) Scattering in Heat Transport Across Graphene/SiO2 Interfaces, Nano Lett, 16(10) (2016) 6014-6020

[65] T. Jiang, X. Zhang, S. Vishwanath, X. Mu, V. Kanzyuba, D.A. Sokolov, S. Ptasinska, D.B. Go, H.G. Xing and T. Luo, Covalent bonding modulated graphene-metal interfacial thermal transport, Nanoscale, 8(21) (2016) 10993-1001

[66] A. Majumdar and P. Reddy, Role of electron-phonon coupling in thermal conductance of metal-nonmetal interfaces, Applied Physics Letters, 84(23) (2004) 4768-4770

[67] H.-K. Lyeo and D.G. Cahill, Thermal conductance of interfaces between highly dissimilar materials, Physical Review B, 73(14) (2006) 144301

[68] C.-C. Chen, Z. Li, L. Shi and S.B. Cronin, Thermal interface conductance across a graphene/hexagonal boron nitride heterojunction, Applied Physics Letters, 104((2014) 081908

[69] J. Judek, A.P. Gertych, M. Swiniarski, A. Lapinska, A. Duzynska and M. Zdrojek, High accuracy determination of the thermal properties of supported 2D materials, Sci Rep, 5((2015) 12422

[70] X. Tang, S. Xu and X. Wang, Corrugated epitaxial graphene/SiC interfaces: photon excitation and probing, Nanoscale, 6((2014) 8822-8830

[71] P. Yuan, C. Li, S. Xu, J. Liu and X. Wang, Interfacial thermal conductance between few to tens of layered-MoS2 and c-Si: Effect of MoS2 thickness, Acta Materialia, 122((2017) 152-165 
[72] T. Beechem, A. Christensen, S. Graham and D. Green, Micro-Raman thermometry in the presence of complex stresses in GaN devices, Journal of Applied Physics, 103(12) (2008) 124501

[73] T.E. Beechem and J.R. Serrano, Raman thermometry of microdevices: Comparing methods to minimize error, (2011)

[74] A.O. Mohamed and S. Deepak, Temperature dependence of the thermal conductivity of single-wall carbon nanotubes, Nanotechnology, 12(1) (2001) 21

[75] Huang .Z, Tang .Z.a, Yu .J and B. .S, Temperature-dependent thermal conductivity of bent carbon nanotubes by molecular dynamics simulation, Journal of Applied Physics, 109(10) (2011) 104316

[76] N. Kondo, T. Yamamoto and K. Watanabe, Molecular-dynamics simulations of thermal transport in carbon nanotubes with structural defects, e-Journal of Surface Science and Nanotechnology, $4((2006)$ 239-243

[77] X. Huang, X. Huai, S. Liang and X. Wang, Thermal transport in Si/Ge nanocomposites, Journal of Physics D: Applied Physics, 42(9) (2009) 095416

[78] J. Zhang and X. Wang, Thermal transport in bent graphene nanoribbons, Nanoscale, 5(2) (2013) 734-743

[79] J. Zhang, X. Wang and H. Xie, Co-existing heat currents in opposite directions in graphene nanoribbons, Physics Letters A, 377(41) (2013) 2970-2978

[80] J. Zhang, Y. Hong and Y. Yue, Thermal transport across graphene and single layer hexagonal boron nitride, Journal of Applied Physics, 117(13) (2015) 134307

[81] Y. Hong, J. Zhang and X.C. Zeng, Interlayer thermal conductance within a phosphorene and graphene bilayer, Nanoscale, 8(46) (2016) 19211-19218

[82] Y. Hong, C. Zhu, M. Ju, J. Zhang and X.C. Zeng, Lateral and flexural phonon thermal transport in graphene and stanene bilayers, Phys Chem Chem Phys, 19(9) (2017) 6554-6562

[83] J. Zhang, Y. Hong, Z. Tong, Z. Xiao, H. Bao and Y. Yue, Molecular dynamics study of interfacial thermal transport between silicene and substrates, Physical Chemistry Chemical Physics, 17(37) (2015) 23704-23710

[84] J. Zhang, Y. Wang and X. Wang, Rough contact is not always bad for interfacial energy coupling, Nanoscale, 5(23) (2013) 11598-11603

[85] Y. Hong, L. Li, X.C. Zeng and J. Zhang, Tuning thermal contact conductance at graphene-copper interface via surface nanoengineering, Nanoscale, 7(14) (2015) 6286-6294

[86] J.D.N. Cheeke, H. Ettinger and B. Hebral, Analysis of heat transfer between solids at low temperatures, Canadian Journal of Physics, 54(17) (1976) 1749-1771

[87] W.A. Little, The Transport of Heat Between Dissimilar Solids at Low Temperatures, (1996) $15-30$

[88] P.E. Hopkins, P.M. Norris, M.S. Tsegaye and A.W. Ghosh, Extracting phonon thermal conductance across atomic junctions: Nonequilibrium Green's function approach compared to semiclassical methods, Journal of Applied Physics, 106(6) (2009) 063503

[89] P.M. Norris, N.Q. Le and C.H. Baker, Tuning Phonon Transport: From Interfaces to Nanostructures, Journal of Heat Transfer, 135(6) (2013) 061604-061604

[90] J.C. Duda, P.M. Norris and P.E. Hopkins, On the Linear Temperature Dependence of Phonon 
Thermal Boundary Conductance in the Classical Limit, Journal of Heat Transfer, 133(7) (2011) 074501-074501

[91] J.C. Duda, C.B. Saltonstall, P.M. Norris and P.E. Hopkins, Assessment and prediction of thermal transport at solid-self-assembled monolayer junctions, The Journal of Chemical Physics, 134(9) (2011) 094704

[92] E.T. Swartz and R.O. Pohl, Thermal boundary resistance, Reviews of Modern Physics, 61(3) (1989) 605-668

[93] E.T. Swartz and R.O. Pohl, Thermal resistance at interfaces, Applied Physics Letters, 51(26) (1987) 2200-2202

[94] P.E. Hopkins, J.C. Duda and P.M. Norris, Anharmonic Phonon Interactions at Interfaces and Contributions to Thermal Boundary Conductance, Journal of Heat Transfer, 133(6) (2011) 062401-062401

[95] C.B. Saltonstall, C.A. Polanco, J.C. Duda, A.W. Ghosh, P.M. Norris and P.E. Hopkins, Effect of interface adhesion and impurity mass on phonon transport at atomic junctions, Journal of Applied Physics, 113(1) (2013) 013516

[96] C.A. Polanco, C.B. Saltonstall, P.M. Norris, P.E. Hopkins and A.W. Ghosh, Impedance Matching of Atomic Thermal Interfaces Using Primitive Block Decomposition, Nanoscale and Microscale Thermophysical Engineering, 17(3) (2013) 263-279

[97] N.Q. Le, J.C. Duda, T.S. English, P.E. Hopkins, T.E. Beechem and P.M. Norris, Strategies for tuning phonon transport in multilayered structures using a mismatch-based particle model, Journal of Applied Physics, 111(8) (2012) 084310

[98] T.S. English, J.C. Duda, J.L. Smoyer, D.A. Jordan, P.M. Norris and L.V. Zhigilei, Enhancing and tuning phonon transport at vibrationally mismatched solid-solid interfaces, Physical Review B, 85(3) (2012) 035438

[99] S. Maruyama, Y. Igarashi, Y. Taniguchi and J. Shiomi, Anisotropic Heat Transfer of Single-Walled Carbon Nanotubes, Journal of Thermal Science and Technology, 1(2) (2006) 138-148

[100] H. Zhong and J.R. Lukes, Interfacial thermal resistance between carbon nanotubes: Molecular dynamics simulations and analytical thermal modeling, Physical Review B, 74(12) (2006) 125403

[101] W.J. Evans, M. Shen and P. Keblinski, Inter-tube thermal conductance in carbon nanotubes arrays and bundles: Effects of contact area and pressure, Applied Physics Letters, 100(26) (2012) 261908

[102] V. Varshney, S.S. Patnaik, A.K. Roy and B.L. Farmer, Modeling of Thermal Conductance at Transverse CNT-CNT Interfaces, The Journal of Physical Chemistry C, 114(39) (2010) 16223-16228 [103] Z. Xu and M.J. Buehler, Nanoengineering Heat Transfer Performance at Carbon Nanotube Interfaces, ACS Nano, 3(9) (2009) 2767-2775

[104] G.-J. Hu and B.-Y. Cao, Thermal resistance between crossed carbon nanotubes: Molecular dynamics simulations and analytical modeling, Journal of Applied Physics, 114(22) (2013) 224308

[105] L. Hu and A.J.H. McGaughey, Thermal conductance of the junction between single-walled carbon nanotubes, Applied Physics Letters, 105(19) (2014) 193104

[106] R.N. Salaway and L.V. Zhigilei, Thermal conductance of carbon nanotube contacts: Molecular dynamics simulations and general description of the contact conductance, Physical Review B, 94(1) 
(2016)

[107] T. Li, Z.A. Tang, Z.X. Huang and J. Yu, Interfacial thermal resistance of 2D and 1D carbon/hexagonal boron nitride van der Waals heterostructures, Carbon, 105((2016) 566-571

[108] J. Wang and H. Xie, Molecular dynamic investigation on the structures and thermal properties of carbon nanotube interfaces, Applied Thermal Engineering, 88((2015) 347-352

[109] W. Chen, J. Zhang and Y. Yue, Molecular dynamics study on thermal transport at carbon nanotube interface junctions: Effects of mechanical force and chemical functionalization, International Journal of Heat and Mass Transfer, 103((2016) 1058-1064

[110] Z.-Y. Ong and E. Pop, Molecular dynamics simulation of thermal boundary conductance between carbon nanotubes and SiO2, Physical Review B, 81(15) (2010) 155408

[111] M. Hu, P. Keblinski, J.-S. Wang and N. Raravikar, Interfacial thermal conductance between silicon and a vertical carbon nanotube, Journal of Applied Physics, 104(8) (2008) 083503

[112] H. Bao, C. Shao, S. Luo and M. Hu, Enhancement of interfacial thermal transport by carbon nanotube-graphene junction, Journal of Applied Physics, 115(5) (2014) 053524

[113] Y. Feng, J. Zhu and D. Tang, Effect of van der Waals forces on thermal conductance at the interface of a single-wall carbon nanotube array and silicon, AIP Advances, 4(12) (2014) 127118

[114] J. Shi, Y. Dong, T. Fisher and X. Ruan, Thermal transport across carbon nanotube-graphene covalent and van der Waals junctions, Journal of Applied Physics, 118(4) (2015) 044302

[115] Y. Ni, H. Han, S. Volz and T. Dumitrică, Nanoscale Azide Polymer Functionalization: A Robust Solution for Suppressing the Carbon Nanotube-Polymer Matrix Thermal Interface Resistance, The Journal of Physical Chemistry C, 119(22) (2015) 12193-12198

[116] V. Samvedi and V. Tomar, Role of Interface Thermal Boundary Resistance, Straining, and Morphology in Thermal Conductivity of a Set of Si-Ge Superlattices and Biomimetic Si-Ge Nanocomposites, Proceedings of the Asme Pacific Rim Technical Conference and Exhibition on Packaging and Integration of Electronic and Photonic Systems, Mems and Nems 2011, Vol 1, (2012) 361-367

[117] X.W. Zhou, R.E. Jones, C.J. Kimmer, J.C. Duda and P.E. Hopkins, Relationship of thermal boundary conductance to structure from an analytical model plus molecular dynamics simulations, Physical Review B, 87(9) (2013) 094303

[118] V. Samvedi and V. Tomar, The role of straining and morphology in thermal conductivity of a set of Si-Ge superlattices and biomimetic Si-Ge nanocomposites, Journal of Physics D-Applied Physics, 43(13) (2010) 135401

[119] S. Murad and I.K. Puri, Communication: Thermal rectification in liquids by manipulating the solid-liquid interface, Journal of Chemical Physics, 137(8) (2012) 081101

[120] M.C. Wang, N. Hu, L.M. Zhou and C. Yan, Enhanced interfacial thermal transport across graphene-polymer interfaces by grafting polymer chains, Carbon, 85((2015) 414-421

[121] T. Kuila, S. Bose, A.K. Mishra, P. Khanra, N.H. Kim and J.H. Lee, Chemical functionalization of graphene and its applications, Progress in Materials Science, 57(7) (2012) 1061-1105

[122] D.W. Boukhvalov and M.I. Katsnelson, Chemical functionalization of graphene, Journal of Physics: Condensed Matter, 21(34) (2009) 344205

[123] A.K. Singh and B.I. Yakobson, Electronics and Magnetism of Patterned Graphene Nanoroads, 
Nano Letters, 9(4) (2009) 1540-1543

[124] H. Zhang, A.F. Fonseca and K. Cho, Tailoring Thermal Transport Property of Graphene through Oxygen Functionalization, The Journal of Physical Chemistry C, 118(3) (2014) 1436-1442

[125] J.Y. Kim, J.-H. Lee and J.C. Grossman, Thermal Transport in Functionalized Graphene, ACS Nano, 6(10) (2012) 9050-9057

[126] J. Zhang, X. He, L. Yang, G. Wu, J. Sha, C. Hou, C. Yin, A. Pan, Z. Li and Y. Liu, Effect of Tensile Strain on Thermal Conductivity in Monolayer Graphene Nanoribbons: A Molecular Dynamics Study, Sensors, 13(7) (2013) 9388

[127] B. Liu, J.A. Baimova, C.D. Reddy, A.W.-K. Law, S.V. Dmitriev, H. Wu and K. Zhou, Interfacial Thermal Conductance of a Silicene/Graphene Bilayer Heterostructure and the Effect of Hydrogenation, ACS Applied Materials \& Interfaces, 6(20) (2014) 18180-18188

[128] S. Xu, T. Wang, D. Hurley, Y. Yue and X. Wang, Development of time-domain differential Raman for transient thermal probing of materials, Optics Express, 23(8) (2015) 10040-10056

[129] T. Wang, S. Xu, D.H. Hurley, Y. Yue and X. Wang, Frequency-resolved Raman for transient thermal probing and thermal diffusivity measurement, Opt Lett, 41(1) (2016) 80-3

[130] C. Li, S. Xu, Y. Yue, B. Yang and X. Wang, Thermal characterization of carbon nanotube fiber by time-domain differential Raman, Carbon, 103((2016) 101-108

[131] S. Kaur, N. Raravikar, B.A. Helms, R. Prasher and D.F. Ogletree, Enhanced thermal transport at covalently functionalized carbon nanotube array interfaces, Nat Commun, 5((2014) 3082

[132] P. Vogt, P. De Padova, C. Quaresima, J. Avila, E. Frantzeskakis, M.C. Asensio, A. Resta, B. Ealet and G. Le Lay, Silicene: compelling experimental evidence for graphenelike two-dimensional silicon, Phys Rev Lett, 108(15) (2012) 155501

[133] L. Song, L. Ci, H. Lu, P.B. Sorokin, C. Jin, J. Ni, A.G. Kvashnin, D.G. Kvashnin, J. Lou, B.I. Yakobson and P.M. Ajayan, Large scale growth and characterization of atomic hexagonal boron nitride layers, Nano Lett, 10(8) (2010) 3209-15

[134] S. Sahoo, A.P.S. Gaur, M. Ahmadi, M.J.F. Guinel and R.S. Katiyar, Temperature-Dependent Raman Studies and Thermal Conductivity of Few-Layer MoS2, The Journal of Physical Chemistry C, 117(17) (2013) 9042-9047

[135] L. Li, Y. Yu, G.J. Ye, Q. Ge, X. Ou, H. Wu, D. Feng, X.H. Chen and Y. Zhang, Black phosphorus field-effect transistors, Nature Nanotechnology, 9(5) (2014) 372-377

[136] H. Li, Q. Zhang, C.C.R. Yap, B.K. Tay, T.H.T. Edwin, A. Olivier and D. Baillargeat, From Bulk to Monolayer MoS2: Evolution of Raman Scattering, Advanced Functional Materials, 22(7) (2012) 1385-1390

[137] A. Splendiani, L. Sun, Y. Zhang, T. Li, J. Kim, C.Y. Chim, G. Galli and F. Wang, Emerging photoluminescence in monolayer MoS2, Nano Lett, 10(4) (2010) 1271-5

[138] A. Taube, J. Judek, A. Łapińska and M. Zdrojek, Temperature-Dependent Thermal Properties of Supported MoS2 Monolayers, ACS Applied Materials \& Interfaces, 7(9) (2015) 5061-5065

[139] Y. Yue, K. Liu, M. Li and X. Hu, Thermal manipulation of carbon nanotube fiber by mechanical stretching, Carbon, 77((2014) 973-979

[140] M. Li, Y. Sun, H. Xiao, X. Hu and Y. Yue, High temperature dependence of thermal transport in graphene foam, Nanotechnology, 26(10) (2015) 105703 
[141] M.T. Pettes, H. Ji, R.S. Ruoff and L. Shi, Thermal Transport in Three-Dimensional Foam Architectures of Few-Layer Graphene and Ultrathin Graphite, Nano Letters, 12(6) (2012) 2959-2964

[142] M. Shen, P.K. Schelling and P. Keblinski, Heat transfer mechanism across few-layer graphene by molecular dynamics, Physical Review B, 88(4) (2013) 045444

[143] A.N. Volkov and L.V. Zhigilei, Scaling laws and mesoscopic modeling of thermal conductivity in carbon nanotube materials, Phys Rev Lett, 104(21) (2010) 215902

[144] Z. Fan, F. Gong, S.T. Nguyen and H.M. Duong, Advanced multifunctional graphene aerogel Poly (methyl methacrylate) composites: Experiments and modeling, Carbon, 81((2015) 396-404

[145] M. Hu, S. Shenogin, P. Keblinski and N. Raravikar, Thermal energy exchange between carbon nanotube and air, Applied Physics Letters, 90(23) (2007) 231905

[146] S. Hida, T. Hori, T. Shiga, J. Elliott and J. Shiomi, Thermal resistance and phonon scattering at the interface between carbon nanotube and amorphous polyethylene, International Journal of Heat and Mass Transfer, 67((2013) 1024-1029

[147] K. Toprak and Y. Bayazitoglu, Interfacial thermal resistance of Cu-SWCNT nanowire in water, International Journal of Heat and Mass Transfer, 79((2014) 584-588

[148] D.J. Rogers, J.M. Qu and M. Yao, Molecular Dynamics Simulation of the Thermal Resistance of Carbon Nanotube - Substrate Interfaces, Ipack 2009: Proceedings of the Asme Interpack Conference 2009, Vol 2, (2010) 55-61

[149] F. Gao, J.M. Qu and M. Yao, Interfacial thermal resistance between metallic carbon nanotube and $\mathrm{Cu}$ substrate, Journal of Applied Physics, 110(12) (2011) 124314

[150] X. Yu, L. Zhang, X. Song, T. Xi, Y. Zhao, J. Liu, X. Yang, M. Chen and P. Yang, Investigation of thermal resistance for the graphene-Si interface by molecular dynamics, Int. J. Mater. Struct. Integr., 6((2012) 65-73

[151] A.K. Vallabhaneni, B. Qiu, J. Hu, Y.P. Chen, A.K. Roy and X. Ruan, Interfacial thermal conductance limit and thermal rectification across vertical carbon nanotube/graphene nanoribbon-silicon interfaces, Journal of Applied Physics, 113(6) (2013) 064311

[152] Z. Xu and M.J. Buehler, Heat dissipation at a graphene-substrate interface, Journal of Physics: Condensed Matter, 24(47) (2012) 475305

[153] Z. Wei, Z. Ni, K. Bi, M. Chen and Y. Chen, Interfacial thermal resistance in multilayer graphene structures, Physics Letters A, 375(8) (2011) 1195-1199

[154] B. Liu, J.A. Baimova, C.D. Reddy, S.V. Dmitriev, W.K. Law, X.Q. Feng and K. Zhou, Interface thermal conductance and rectification in hybrid graphene/silicene monolayer, Carbon, 79((2014) 236-244

[155] Z. Ding, Q.-X. Pei, J.-W. Jiang, W. Huang and Y.-W. Zhang, Interfacial thermal conductance in graphene/MoS2 heterostructures, Carbon, 96((2016) 888-896

[156] B. Liu, F. Meng, C.D. Reddy, J.A. Baimova, N. Srikanth, S.V. Dmitriev and K. Zhou, Thermal transport in a graphene-MoS2 bilayer heterostructure: a molecular dynamics study, RSC Advances, 5(37) (2015) 29193-29200

[157] L. Hu, T. Desai and P. Keblinski, Determination of interfacial thermal resistance at the nanoscale, Physical Review B, 83(19) (2011) 195423

[158] Y. Hong, J. Zhang and X.C. Zeng, Thermal contact resistance across a linear heterojunction 
within a hybrid graphene/hexagonal boron nitride sheet, Physical Chemistry Chemical Physics, 18((2016) 24164-24170

[159] J. Zhang, Y. Hong, M. Liu, Y. Yue, Q. Xiong and G. Lorenzini, Molecular dynamics simulation of the interfacial thermal resistance between phosphorene and silicon substrate, International Journal of Heat and Mass Transfer, 104((2017) 871-877 


\section{List of Tables and Figures}

Table 1. Summary of experimental results of interfacial thermal transport of atomic-layer materials

Table 2. Summary of MD simulation results of interfacial thermal transport of atomic-layer materials.

Figure 1. (a) Thermal contact resistance measurement of CNT-CNTs by using micro-bridge method [21]. (b) Contact transient electro-thermal (CTET) technique to measure thermal contact resistance (point-contact) between microwires [31]. (c) The principle of 1-D thermal contact resistance measurement between a lateral CNT and substrate [33]. (d) Schematic of thermal contact resistance analysis of aligned TiO2 arrays [37]. (e) FDTR experimental setup and sample configuration [46]. (f) Schematic of characterizing sandwiched structure of $\mathrm{Al} /$ graphene/Si interface by using TDTR (ultra-fast pump-probe) method [25].

Figure 2. (a) The schematic of electrical heating Raman probing method for measuring graphene interface thermal transport[14]. (b) Schematic of experimental setup for characterization of the graphene/SiC interface based on photon-excitation and Raman probing [70].

Figure 3. Thermal transport involved in ultrafast pump probe thermal characterizations: absorbed photon energy is firstly transferred to the electrons. Energy is coupled through the electron scattering and the electron-phonon energy exchange in metal layer. At the metal layer/graphene interface, there are boundary scattering, the phonon-phonon energy coupling, and the electron-phonon energy exchange between metal layer and graphene.

Figure 4. Schematic of showing the Raman intensity enhancement due to air spacing between graphene and substrate as a result of heating measurement [70]. (a) There is structural derivation between calibration and measurement 
experiments. (b) It reveals that interference effect due to the little spacing (nanoscale) can improve the Raman excitation significantly. (c) The relationship between the enhancement factor and thickness of air layer.

Figure 5. (a) The heat conduction through two aligned CNTs with one ends overlapping and the other ends setting as the hot and cold spots respectively [100]. (with permission from American Physical Society) (b) Atomic configuration of two parallel CNTs connected with hydrocarbon $\mathrm{CH}_{2}$ chain linkers [109]. (c) 3D CNT-graphene structure: The pillared graphene structure with covalent bonds at junctions [114]. (with permission from American Physical Society)

Figure 6. (a) Interatomic force distributions in the GNR system at out-of-plane (z) direction. Significant attractive and repulsive forces are detected in the suspended and supported regions, respectively. (b) Radial distribution function between GNR and copper for different $\delta$ cases. A sudden separation between GNR and copper atoms is observed when $\delta$ increases from $0.63 \mathrm{~nm}$ to $0.83 \mathrm{~nm}$ [85]. (with permission from Royal Society of Chemistry) 
Table 1. Summary of experimental results of interfacial thermal transport of atomic-layer materials.

\begin{tabular}{|c|c|c|c|}
\hline Interface & Thermal resistance /conductance & Method & Ref. \\
\hline $\mathrm{CNT} / \mathrm{CNT}$ & $\begin{array}{l}1.2 \times 10^{-8} \mathrm{~K} \cdot \mathrm{m}^{2} / \mathrm{W} \text { (aligned); } \\
1.22 \times 10^{-9} \mathrm{~K} \cdot \mathrm{m}^{2} / \mathrm{W} \text { (cross) }\end{array}$ & Microbridge & [21] \\
\hline $\mathrm{CNT} / \mathrm{CNT}$ & $\begin{array}{l}3 \times 10^{8}-1.3 \times 10^{9} \mathrm{~W} / \mathrm{m}^{2} \cdot \mathrm{K} \\
(\text { cross-contact) }\end{array}$ & Microbridge & [22] \\
\hline $\mathrm{Pt} / \mathrm{Pt}$ microwire & $10^{-7}-10^{-5} \mathrm{~K} \cdot \mathrm{m}^{2} / \mathrm{W}$ & CTET & {$[31]$} \\
\hline $\mathrm{CNT} /$ substrate & $1-2 \times 10^{-8} \mathrm{~K} \cdot \mathrm{m}^{2} / \mathrm{W}$ & Electrical heating & {$[33]$} \\
\hline $\mathrm{CNT} /$ substrate & $3 \mathrm{~K} \cdot \mathrm{m} / \mathrm{W}$ & Electrical heating & {$[62]$} \\
\hline $\mathrm{TiO}_{2}$ nanotubes & $\begin{array}{l}15.1 \mathrm{~K} \cdot \mathrm{m}^{2} / \mathrm{W} \\
20.6 \mathrm{~K} \cdot \mathrm{m}^{2} / \mathrm{W} \text { (amorphous) } \\
5.90 \mathrm{~K} \cdot \mathrm{m}^{2} / \mathrm{W} \\
\text { (anatase) }\end{array}$ & TET & [37] \\
\hline CNT/substrate & $0.007-0.06 \mathrm{~W} / \mathrm{m} \cdot \mathrm{K}$ & Electrical heating & [35] \\
\hline $\mathrm{Al} /$ graphene/SiO${ }_{2}$ & $5.6 \times 10^{-9}$ to $1.2 \times 10^{-8} \mathrm{~K} \cdot \mathrm{m}^{2} / \mathrm{W}$ & $3 \omega$ & {$[24]$} \\
\hline $\mathrm{Al} /$ graphene/Si & $6.2 \times 10^{7} \mathrm{~W} / \mathrm{m}^{2} \cdot \mathrm{K}$ & TDTR & {$[25]$} \\
\hline $\mathrm{Au} / \mathrm{Ti} /$ graphene/ $\mathrm{SiO}_{2}$ & $2.5 \times 10^{7} \mathrm{~W} / \mathrm{m}^{2} \cdot \mathrm{K}$ & TDTR & {$[56]$} \\
\hline $\mathrm{Al} /$ graphene/ $\mathrm{SiO}_{2}$ & $2-3 \times 10^{7} \mathrm{~W} / \mathrm{m}^{2} \cdot \mathrm{K}$ & TDTR & [39] \\
\hline $\mathrm{Au} / \mathrm{O} /$ graphene & $2.433-3.241 \times 10^{7} \mathrm{~W} / \mathrm{m}^{2} \cdot \mathrm{K}$ & TDTR & {$[63]$} \\
\hline $\mathrm{Au} / \mathrm{N} / \mathrm{graphene}$ & $2.141-2.898 \times 10^{7} \mathrm{~W} / \mathrm{m}^{2} \cdot \mathrm{K}$ & IDTK & [03] \\
\hline $\mathrm{Al} /$ graphene/ $\mathrm{SiO}_{2}$ & $2-3 \times 10^{7} \mathrm{~W} / \mathrm{m}^{2} \cdot \mathrm{K}$ & TDTR & [39] \\
\hline Graphene/SiO${ }_{2}$ & $5 \times 10^{7} \mathrm{~W} / \mathrm{m}^{2} \cdot \mathrm{K}$ & TDTR & {$[55]$} \\
\hline $\mathrm{SiO}_{2} /$ graphene $/ \mathrm{SiO}_{2}$ & $4.1 \times 10^{7} \mathrm{~W} / \mathrm{m}^{2} \cdot \mathrm{K}$ & TDTR & {$[64]$} \\
\hline Graphene $/ \mathrm{SiO}_{2}$ & $4.2 \times 10^{7} \mathrm{~W} / \mathrm{m}^{2} \cdot \mathrm{K}$ & FDTR & [46] \\
\hline Au/alkanedithiol/Au & $6.5 \times 10^{7} \mathrm{~W} / \mathrm{m}^{2} \cdot \mathrm{K}$ & FDTR & [47] \\
\hline Au/alkanedithiol/Pd & $3.6 \times 10^{7} \mathrm{~W} / \mathrm{m}^{2} \cdot \mathrm{K}$ & & \\
\hline $\mathrm{Au} / \mathrm{Gr} / \mathrm{Al}_{2} \mathrm{O}_{3}$ & $1.8 \times 10^{8} \mathrm{~W} / \mathrm{m}^{2} \cdot \mathrm{K}$ & FDTR & [48] \\
\hline $\mathrm{Au} / \mathrm{Cu} / \mathrm{Al}_{2} \mathrm{O}_{3}$ & $3.9 \times 10^{8} \mathrm{~W} / \mathrm{m}^{2} \cdot \mathrm{K}$ & & \\
\hline Graphene/SiC & $5.3 \times 10^{-5} \mathrm{~K} \cdot \mathrm{m}^{2} / \mathrm{W}$ & $\begin{array}{l}\text { Electrical-heating } \\
\text { Raman-probing }\end{array}$ & [14] \\
\hline Graphene/h-BN & $7.41 \times 10^{6} \mathrm{~W} / \mathrm{m}^{2} \cdot \mathrm{K}$ & $\begin{array}{l}\text { Electrical-heating } \\
\text { Raman-probing }\end{array}$ & {$[68]$} \\
\hline Graphene/Si & $2.8 \times 10^{7} \mathrm{~W} / \mathrm{m}^{2} \cdot \mathrm{K}$ & Photon-heating Raman-probing & {$[61]$} \\
\hline Graphene/Si & $183 \mathrm{~W} / \mathrm{m}^{2} \cdot \mathrm{K}$ & Photon-heating Raman-probing & [26] \\
\hline Graphene $/ \mathrm{SiO}_{2}$ & $266 \mathrm{~W} / \mathrm{m}^{2} \cdot \mathrm{K}$ & Photon-heating Raman-probing & [26] \\
\hline Graphene/SiC & $410 \mathrm{~W} / \mathrm{m}^{2} \cdot \mathrm{K}$ & Photon-heating Raman-probing & {$[70]$} \\
\hline Graphene $/ \mathrm{SiO}_{2} / \mathrm{Si}$ & $1.7 \times 10^{6} \mathrm{~W} / \mathrm{m}^{2} \cdot \mathrm{K}$ & Raman-probing & [69] \\
\hline $\mathrm{MoS}_{2} / \mathrm{SiO}_{2}$ & $1.25-1.94 \times 10^{6} \mathrm{~W} / \mathrm{m}^{2} \cdot \mathrm{K}$ & Photon-heating Raman-probing & [138] \\
\hline
\end{tabular}


Table 2. Summary of MD simulation results of interfacial thermal transport of atomic-layer materials.

\begin{tabular}{|c|c|c|c|}
\hline Interface & Thermal resistance /conductance & Temperature & Ref. \\
\hline CNT/CNT & $4.04 \times 10^{6} \mathrm{~W} / \mathrm{m}^{2} \cdot \mathrm{K}$ & $300 \mathrm{~K}$ & [99] \\
\hline $\mathrm{CNT} / \mathrm{CNT}$ & $1.1 \times 10^{-7} \mathrm{Km}^{2} / \mathrm{W}$ & $300 \mathrm{~K}$ & {$[100]$} \\
\hline $\mathrm{CNT} / \mathrm{CNT}$ & $1 \times 10^{8}-1 \times 10^{9} \mathrm{~W} / \mathrm{m}^{2} \cdot \mathrm{K}$ & $300 \mathrm{~K}$ & {$[103]$} \\
\hline $\mathrm{CNT} / \mathrm{CNT}$ & $4 \times 10^{-11} \mathrm{~W} / \mathrm{K}$ & $300 \mathrm{~K}$ & {$[105]$} \\
\hline CNT/BNNT & $10^{-7} \sim 10^{-6} \mathrm{~K} \cdot \mathrm{m}^{2} / \mathrm{W}$ & $200 \sim 600 \mathrm{~K}$ & [107] \\
\hline $\mathrm{CNT} / \mathrm{CNT}$ & $1.25 \times 10^{-6} \mathrm{~K} \cdot \mathrm{m}^{2} / \mathrm{W}$ & $200 V$ & {$[100]$} \\
\hline $\mathrm{CNT} / \mathrm{Cu}$ & $6.4 \times 10^{-8} \mathrm{~K} \cdot \mathrm{m}^{2} / \mathrm{W}$ & $300 \mathrm{~K}$ & [108] \\
\hline $\mathrm{CNT} / \mathrm{CNT}$ & $\sim 2.8 \times 10^{-12} \mathrm{~W} / \mathrm{K}$ & $300 \mathrm{~K}$ & [109] \\
\hline CNT/graphene & $6 \times 10^{-11} \mathrm{~K} \cdot \mathrm{m}^{2} / \mathrm{W}$ (covalent) & & \\
\hline CNT/graphene & $4 \times 10^{-8} \mathrm{~K} \cdot \mathrm{m}^{2} / \mathrm{W}$ (van der Waals) & $300 \mathrm{~K}$ & [114] \\
\hline CNT-HLK5/ polystyrene & $7 \times 10^{-9} \mathrm{~K} \cdot \mathrm{m}^{2} / \mathrm{W}$ & & \\
\hline CNT-HLK5/ epoxy & $6.6 \times 10^{-9} \mathrm{~K} \cdot \mathrm{m}^{2} / \mathrm{W}$ & $300 \mathrm{~K}$ & {$[115]$} \\
\hline CNT-HLK5/ polyethylene & $6.6 \times 10^{-9} \mathrm{~K} \cdot \mathrm{m}^{2} / \mathrm{W}$ & & \\
\hline CNT/air & $1 \times 10^{5} \mathrm{~W} / \mathrm{m}^{2} \cdot \mathrm{K}$ & $300 \mathrm{~K}$ & {$[145]$} \\
\hline $\mathrm{CNT} /$ polyethylene & $\sim 1 \times 10^{-7}$ & $300 \mathrm{~K} \sim 500 \mathrm{~K}$ & [146] \\
\hline $\mathrm{CNT} /$ water & $3.16 \times 10^{-8}$ & $300 \mathrm{~K}$ & [147] \\
\hline CNT/copper & $\sim 2.5 \times 10^{8} \mathrm{~W} / \mathrm{m}^{2} \cdot \mathrm{K}$ & $300 \mathrm{~K}$ & {$[148]$} \\
\hline CNT/copper & $3.19 \times 10^{-9}$ & $300 \mathrm{~K}$ & [149] \\
\hline CNT/silicon & $5 \times 10^{6} \mathrm{~W} / \mathrm{m}^{2} \cdot \mathrm{K}$ & $300 \mathrm{~K}$ & [111] \\
\hline Graphene/silicon & $3.1 \sim 4.9 \times 10^{-8}$ & $300 \mathrm{~K}$ & [84] \\
\hline Graphene/silicon & $7.5 \times 10^{-8}$ & $300 \mathrm{~K}$ & {$[150]$} \\
\hline Graphene/silicon & $\sim 2 \times 10^{7} \mathrm{~W} / \mathrm{m}^{2} \cdot \mathrm{K}$ & $300 \mathrm{~K}$ & {$[142]$} \\
\hline Graphene/silicon & $1.18-1.47 \times 10^{9} \mathrm{~W} / \mathrm{m}^{2} \cdot \mathrm{K}$ & $300 \mathrm{~K}$ & {$[151]$} \\
\hline Graphene/copper & $2.61 \times 10^{-8}$ & $300 \mathrm{~K}$ & {$[85]$} \\
\hline Graphene/SiC & $1-5 \times 10^{7} \mathrm{~W} / \mathrm{m}^{2} \cdot \mathrm{K}$ & $300 \mathrm{~K}$ & {$[152]$} \\
\hline Graphene/SiC & $7.01 \times 10^{-10}$ & $300 \mathrm{~K}$ & {$[14]$} \\
\hline Graphene/ graphene & $1.48 \sim 4.88 \times 10^{-11}$ & $300 \mathrm{~K}$ & {$[78]$} \\
\hline Graphene/ graphene & $0.2 \sim 4 \times 10^{-9}$ & $300 \mathrm{~K}$ & {$[153]$} \\
\hline Graphene/silicene & $\sim 2.5 \times 10^{8} \mathrm{~W} / \mathrm{m}^{2} \cdot \mathrm{K}$ & $150 \mathrm{~K} \sim 700 \mathrm{~K}$ & {$[154]$} \\
\hline Graphene/silicene & $7 \times 10^{6}-2.2 \times 10^{7} \mathrm{~W} / \mathrm{m}^{2} \cdot \mathrm{K}$ & $200 \mathrm{~K} \sim 700 \mathrm{~K}$ & [139] \\
\hline Graphene/MoS ${ }_{2}$ & $1.38 \times 10^{7} \mathrm{~W} / \mathrm{m}^{2} \cdot \mathrm{K}$ & $300 \mathrm{~K}$ & {$[155]$} \\
\hline Graphene/MoS ${ }_{2}$ & $5.81 \times 10^{6} \mathrm{~W} / \mathrm{m}^{2} \cdot \mathrm{K}$ & $200 \mathrm{~K} \sim 500 \mathrm{~K}$ & {$[156]$} \\
\hline Graphene/resin & $2.1 \times 10^{7} \mathrm{~W} / \mathrm{m}^{2} \cdot \mathrm{K}$ & $300 \mathrm{~K}$ & {$[157]$} \\
\hline Graphene/ $h$-BN & $9.04 \times 10^{-8} \sim 2.96 \times 10^{-7}$ & $200 \mathrm{~K} \sim 700 \mathrm{~K}$ & {$[80]$} \\
\hline Graphene/h-BN & $2.4 \sim 4.1 \times 10^{-10}$ & $200 \mathrm{~K} \sim 600 \mathrm{~K}$ & {$[158]$} \\
\hline Silicene/silicon & $\sim 1.888 \times 10^{-8}$ & $100 \mathrm{~K} \sim 400 \mathrm{~K}$ & [83] \\
\hline
\end{tabular}




\begin{tabular}{l|l|l|l}
\hline Silicene/ amorphous silicon & $\sim 1.342 \times 10^{-8}$ & $100 \mathrm{~K} \sim 400 \mathrm{~K}$ & {$[83]$} \\
Silicene/silica & $\sim 1.366 \times 10^{-8}$ & $100 \mathrm{~K} \sim 400 \mathrm{~K}$ & {$[83]$} \\
Silicene/ amorphous silica & $\sim 1.302 \times 10^{-8}$ & $100 \mathrm{~K} \sim 400 \mathrm{~K}$ & {$[83]$} \\
Phosphorene/ silicon & $1.25 \sim 2.27 \times 10^{-8}$ & $150 \mathrm{~K} \sim 400 \mathrm{~K}$ & {$[159]$} \\
\hline
\end{tabular}



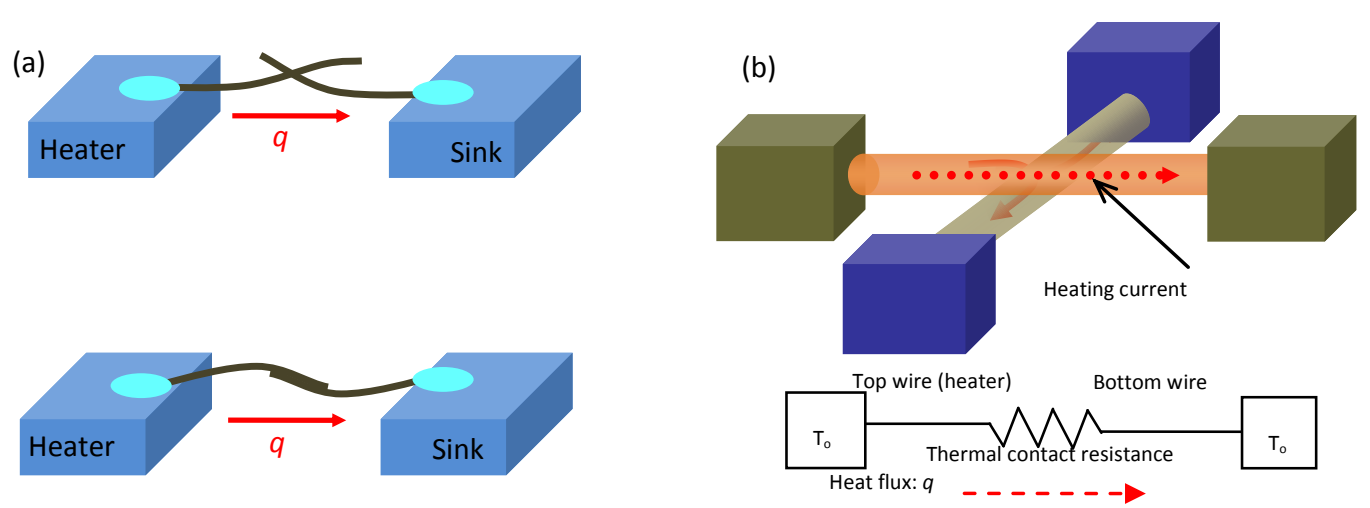

(c)
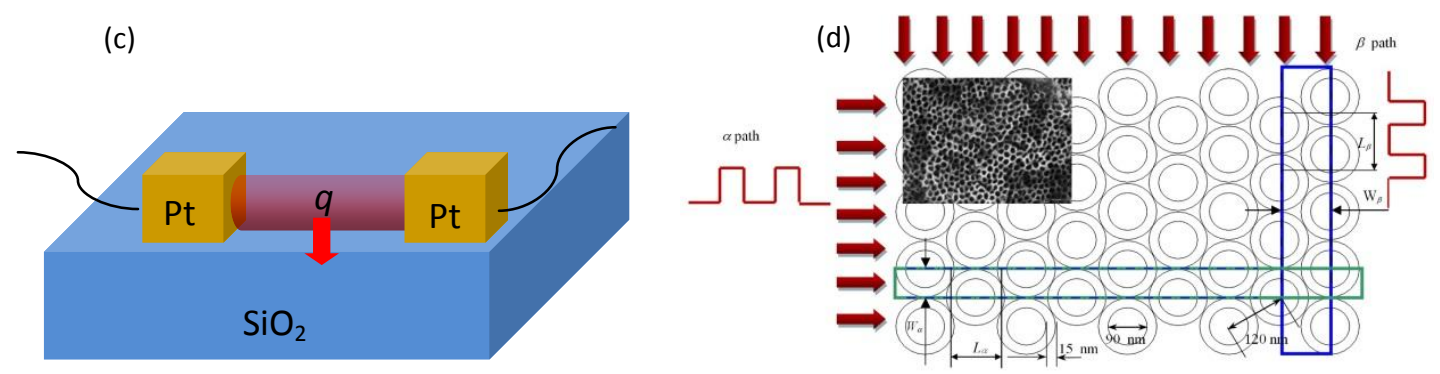

(e)
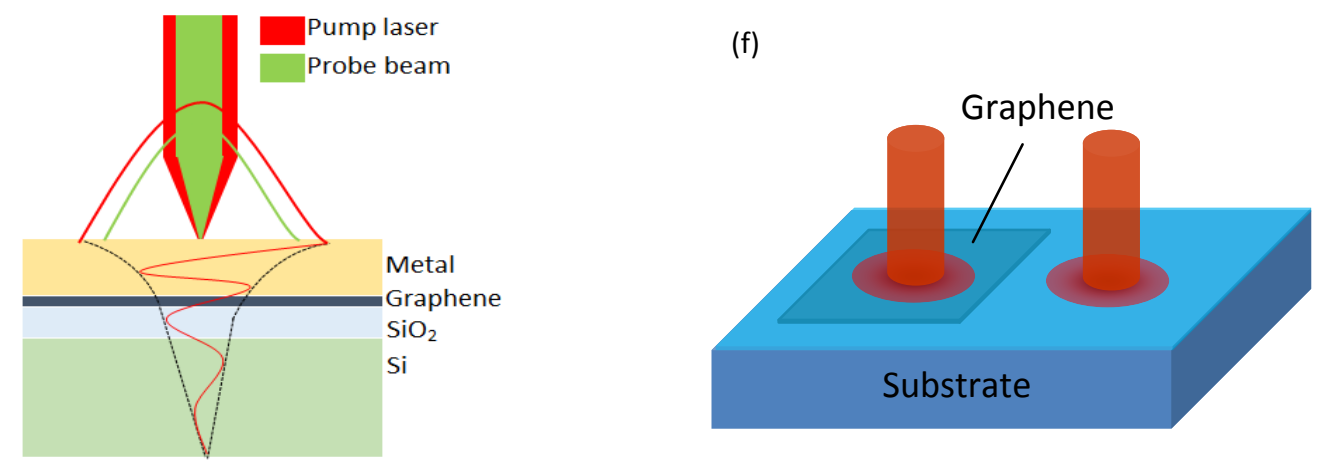

Figure 1(a) Thermal contact resistance measurement of CNT-CNTs by using micro-bridge method [21]. (b) Contact transient electro-thermal (CTET) technique to measure thermal contact resistance (point-contact) between microwires [31]. (c) 1D thermal contact resistance measurement between a lateral CNT and substrate [33]. (d) Thermal contact resistance measurement of aligned $\mathrm{TiO}_{2}$ arrays [37]. (e) FDTR experimental setup and sample configuration [46]. (f) Schematic of characterizing sandwiched structure of Al/graphene/Si interface by using TDTR method [25]. 

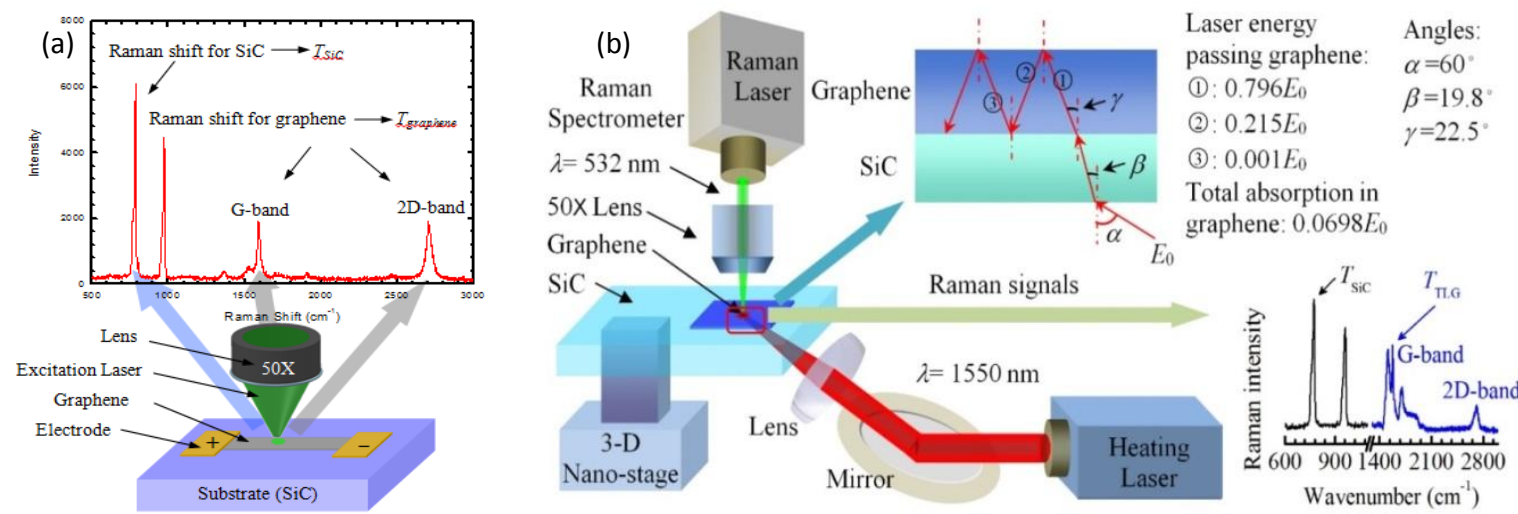

Figure 2. (a) The schematic of electrical heating Raman probing method for measuring graphene interface thermal transport[14]. (b) Schematic of experimental setup for characterization of the graphene/SiC interface based on photon-excitation and Raman probing [70]. 


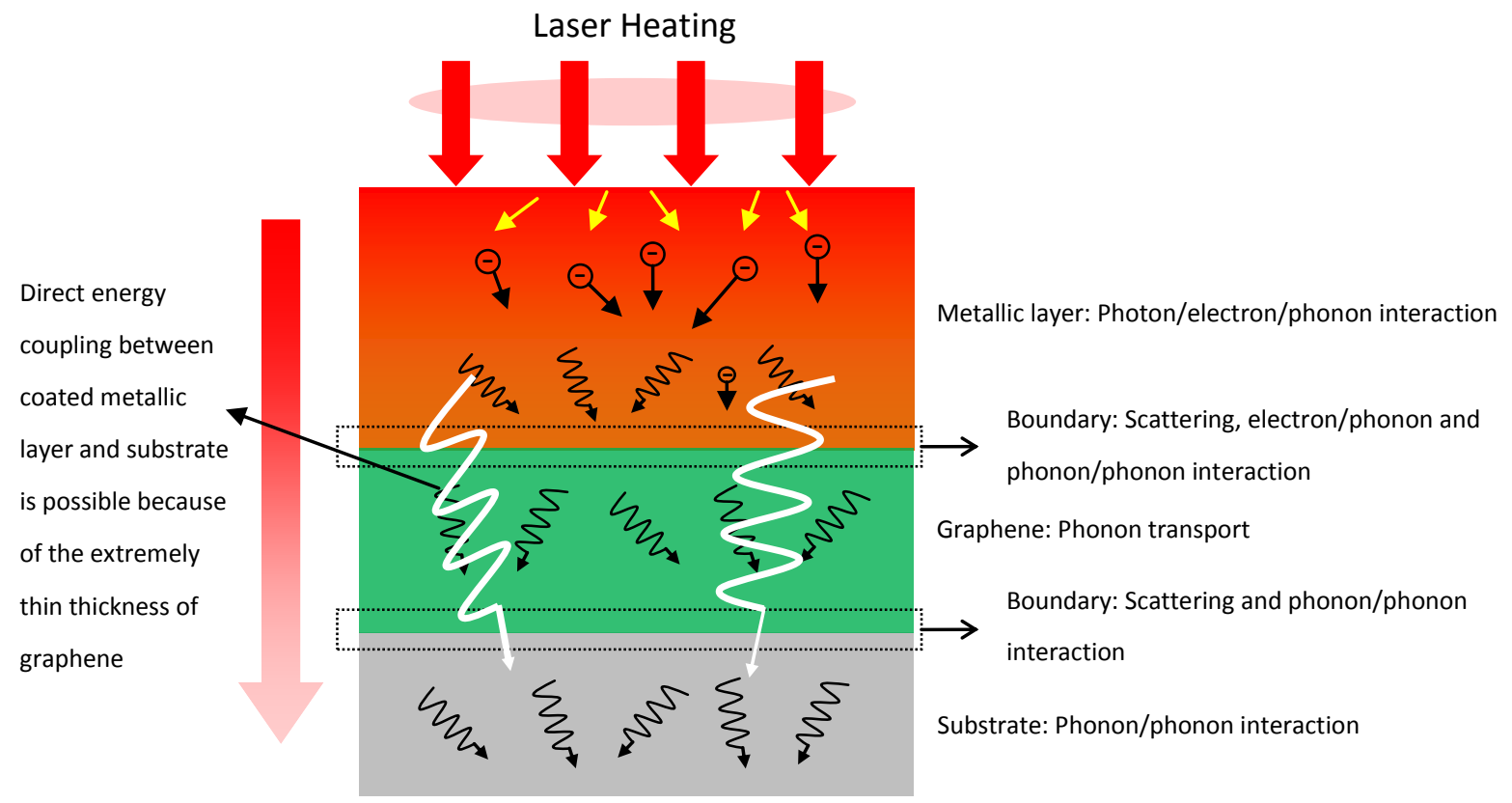

Figure 3. Thermal transport involved in ultrafast pump probe thermal characterizations: absorbed photon energy is firstly transferred to the electrons. Energy is coupled through the electron scattering and the electron-phonon energy exchange in metal layer. At the metal layer/graphene interface, there are boundary scattering, phonon-phonon energy coupling, and electron-phonon energy exchange between metal layer and graphene. 

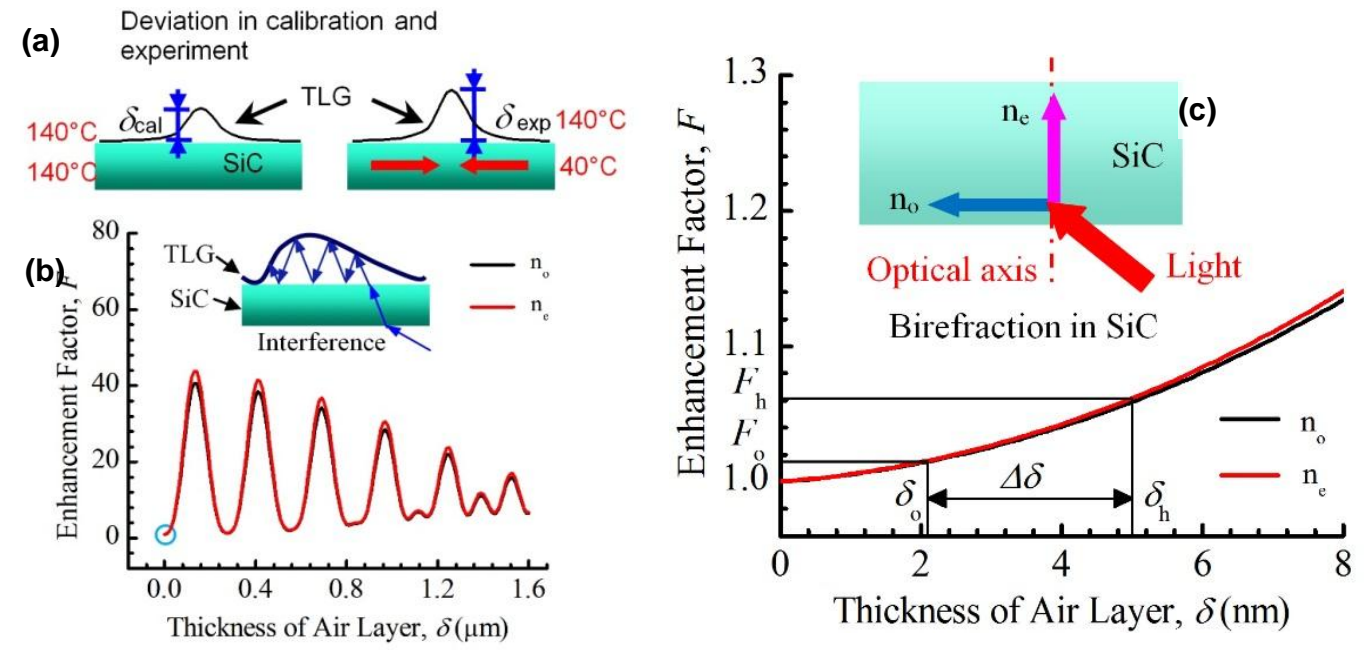

Figure 4. Schematic showing the Raman intensity enhancement due to air spacing between graphene and substrate [70]. (a) There is structural derivation between calibration and measurement experiments. (b) It reveals that interference effect due to little spacing (nanoscale) can improve the Raman excitation significantly. (c) The relationship between enhancement factor and thickness of air layer. 

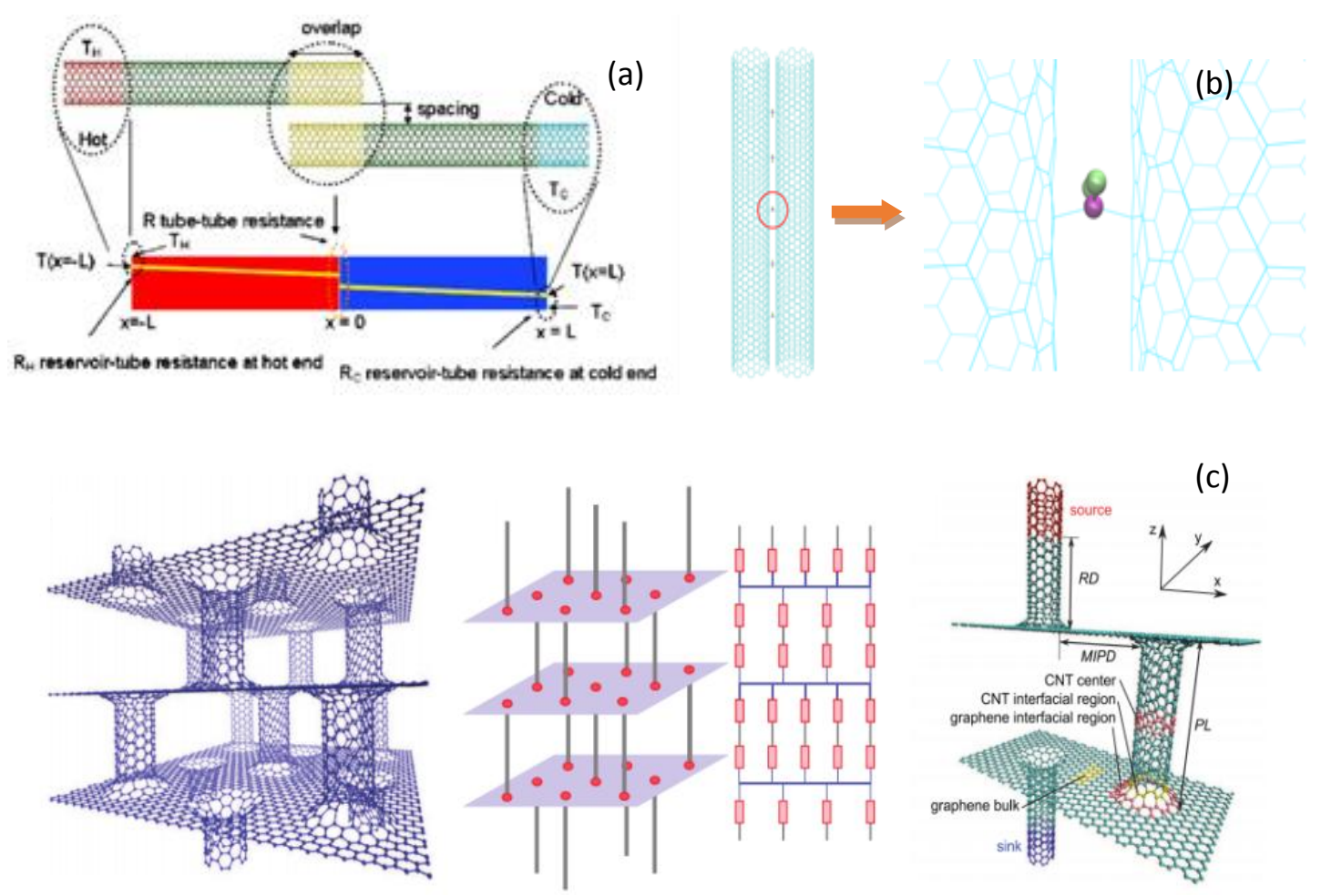

Figure 5. (a) The heat conduction through two aligned CNTs with one ends overlapping and the other ends setting as the hot and cold spots respectively [100]. (with permission from American Physical Society) (b) Atomic configuration of two parallel CNTs connected with hydrocarbon $\mathrm{CH} 2$ chain linkers [109]. (c) 3D CNT-graphene structure: The pillared graphene structure with covalent bonds at junctions [114]. (with permission from American Physical Society) 

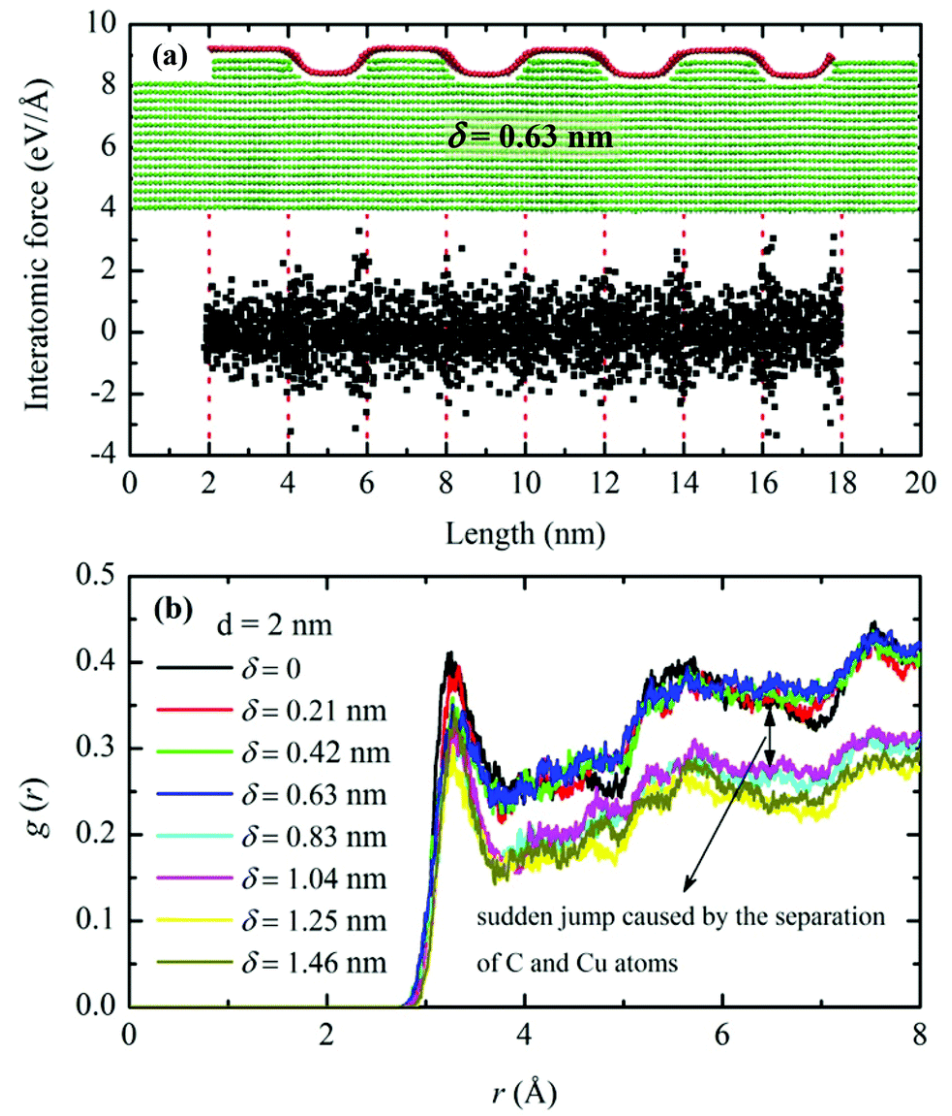

Figure 6. (a) Interatomic force distributions in the GNR system at out-of-plane (z) direction. Significant attractive and repulsive forces are detected in the suspended and supported regions, respectively. (b) Radial distribution function between GNR and copper for different $\delta$ cases. A sudden separation between GNR and copper atoms is observed when $\delta$ increases from $0.63 \mathrm{~nm}$ to $0.83 \mathrm{~nm}$ [85]. (with permission from Royal Society of Chemistry) 